Publ. RIMS, Kyoto Univ.

15 (1979), 653-678

\title{
Propagation of Singularities of Fundamental Solutions of Hyperbolic Mixed Problems
}

By

Seiichiro WAKABAYASHI*

\section{$\S$ 1. Introduction}

In this paper we shall deal with hyperbolic mixed problems with constant coefficients in a quarter-space and study the wave front sets of the fundamental solutions under the only assumption that the hyperbolic mixed problems are $\mathcal{E}$-well posed. Recently Garnir has studied the wave front sets of fundamental solutions for hyperbolic systems [2]. The author was stimulated by his work. For the detailed literatures we refer the reader to [7], [8].

Now let us state our problems, assumptions and main results. Let $\mathbb{R}^{n}$ denote the $n$-dimensional euclidean space and write $x^{\prime}=\left(x_{1}, \cdots, x_{n-1}\right)$ for the coordinate $x=\left(x_{1}, \cdots, x_{n}\right)$ in $\mathbb{R}^{n}$ and $\tilde{\xi}^{\prime}=\left(\xi_{1}, \cdots, \xi_{n-1}\right), \widetilde{\xi}=\left(\xi, \xi_{n+1}\right)$ for the dual coordinate $\xi=\left(\xi_{1}, \cdots, \xi_{n}\right)$. We shall also denote by $\mathbb{R}_{+}^{n}$ the half-space $\left\{x=\left(x^{\prime}, x_{n}\right) \in \mathbb{R}^{n} ; x_{n}>0\right\}$. For differentiation we will use the symbol $D=i^{-1}\left(\partial / \partial x_{1}, \cdots, \partial / \partial x_{n}\right)$. Let $P=P(\xi)$ be a hyperbolic polynomial of order $m$ of $n$ variables $\xi$ with respect to $\vartheta=(1,0, \cdots, 0) \in \mathbb{R}^{n}$ in the sense of Garding, i.e.

$$
P^{0}(-i \vartheta) \neq 0 \text { and } P(\xi-s \vartheta) \neq 0 \text { when } \xi \text { is real and } \operatorname{Im} s<\gamma_{0}
$$

where $P^{0}$ denotes the principal part of $P$, i.e.

$$
P(t \xi)=t^{m}\left(P^{0}(\xi)+o(1)\right) \text { as } t \rightarrow \infty, P^{0}(\xi) \neq \equiv .
$$

Let $\Gamma=\Gamma(P, \vartheta)\left(\subset \mathbb{R}^{n}\right)$ be the component of the set $\left\{\xi \in \mathbb{R}^{n} ; P^{0}(-i \xi) \neq\right.$ 0) which contains $\vartheta$. We also write $\Gamma(P)=\Gamma(P, \vartheta)$. Put

$$
\begin{aligned}
& \Gamma_{0}=\left\{\xi^{\prime} \in \mathbb{R}^{n-1} ; \quad\left(\xi^{\prime}, 0\right) \in \Gamma\right\}, \\
& \dot{\Gamma}=\left\{\xi^{\prime} \in \mathbb{R}^{n-1} ; \quad\left(\xi^{\prime}, \xi_{n}\right) \in \Gamma \text { for some } \xi_{n} \in \mathbb{R}\right\} .
\end{aligned}
$$

Communicated by S. Matsuura, December 23, 1976.

* Institute of Mathematics, the University of Tsukuba. 
The localization $P_{\xi^{0}}(\eta)$ of $P(\xi)$ at $\xi^{0}$ and the multiplicity $m_{\xi^{\circ}}$ of $\xi^{0}$ relative to $P$ are defined by

$$
\nu^{m} P\left(\nu^{-1} \xi^{0}+\eta\right)=\nu^{m \xi^{0}}\left(P_{\xi^{0}}(\eta)+o(1)\right) \text { as } \nu_{\downarrow}^{1} 0, P_{\xi^{0}}(\eta) \not \equiv 0
$$

(see [1]). We note that

$$
\Gamma \subset \Gamma_{\xi^{0} 0} \equiv \Gamma\left(P_{\xi^{0}}\right) .
$$

Now write

$$
P(\xi)=\sum_{j=0}^{m^{\prime}} P_{j}\left(\xi^{\prime}\right) \xi_{n}^{j}, \quad P_{m^{\prime}}\left(\xi^{\prime}\right) \not \equiv 0
$$

Then we see that

$$
P_{m^{\prime}}\left(\xi^{\prime}\right) \neq 0 \text { for } \xi^{\prime} \in \mathbb{R}^{n-1}-i \gamma_{0} \vartheta^{\prime}-i \dot{\Gamma}_{(0,1)} .
$$

In fact, $P_{m^{\prime}}\left(\xi^{\prime}\right)=P_{(0,1)}(\xi)$ and $\Gamma_{(0,1)}=\dot{\Gamma}_{(0,1)} \times \mathbb{R}$. It easily follows that $\Gamma_{0} \subset \dot{\Gamma} \subset \dot{\Gamma}_{(0,1)}$. When $\xi^{\prime} \in \mathbb{R}^{n-1}-i \gamma_{0} \vartheta^{\prime}-i \Gamma_{0}$, we can denote the roots of $P\left(\xi^{\prime}, \lambda\right)=0$ with respect to $\lambda$ by $\lambda_{1}^{+}\left(\xi^{\prime}\right), \cdots, \lambda_{l}^{+}\left(\xi^{\prime}\right), \lambda_{1}^{-}\left(\xi^{\prime}\right), \cdots, \lambda_{m^{\prime}-l}^{-}\left(\xi^{\prime}\right)$, which are enumerated so that $\operatorname{Im} \lambda_{\bar{k}}\left(\xi^{\prime}\right) \gtrless 0$. We consider the mixed initial-boundary value problem for the hyperbolic operator $P(D)$ in a quarter-space

$$
\begin{array}{ll}
P(D) u(x)=f(x), \quad x \in \mathbb{R}_{+}^{n}, & x_{1}>0, \\
\left.D_{1}^{k} u(x)\right|_{x_{1}=0}=0, \quad 0 \leq k \leq m-1, & x_{n}>0, \\
\left.B_{j}(D) u(x)\right|_{x_{n}=0}=0, \quad 1 \leq j \leq l, & x_{1}>0 .
\end{array}
$$

Here the $B_{j}(D)$ are boundary operators with constant coefficients. Put

$$
P_{+}\left(\xi^{\prime}, \lambda\right)=\prod_{j=1}^{l}\left(\lambda-\lambda_{j}^{+}\left(\xi^{\prime}\right)\right), \quad \xi^{\prime} \in \mathbb{R}^{n-1}-i \gamma_{0} \vartheta^{\prime}-i \Gamma_{0} .
$$

Then Lopatinski's determinant for the system $\left\{P, B_{j}\right\}$ is defined by

$$
R\left(\xi^{\prime}\right)=\operatorname{det} L\left(\xi^{\prime}\right) \quad \text { for } \xi^{\prime} \in \boldsymbol{R}^{n-1}-i \gamma_{0} \vartheta^{\prime}-i \Gamma_{0},
$$

where

$$
L\left(\xi^{\prime}\right)=\left(\frac{1}{2 \pi i} \oint B_{j}\left(\xi^{\prime}, \lambda\right) \lambda^{k-1} P_{+}\left(\xi^{\prime}, \lambda\right)^{-1} d \lambda\right)_{j, k=1, \ldots, l}
$$

We impose the following assumption on $\left\{P, B_{j}\right\}$ :

(A) The system $\left\{P, B_{j}\right\}$ is $\mathcal{E}$-well posed, i.e.

$$
R^{0}\left(-i \vartheta^{\prime}\right) \neq 0, R\left(\xi^{\prime}+s \vartheta^{\prime}\right) \neq 0 \text { when } \xi^{\prime} \text { is real and } \operatorname{Im} s<-r_{1} \text {, }
$$


where $R^{0}\left(\xi^{\prime}\right)$ denotes the principal part of $R\left(\xi^{\prime}\right)$ and $\gamma_{1}>\gamma_{0}$ (see [3]).

Now we can construct the fundamental solution $G(x, y)$ for $\left\{P, B_{j}\right\}$ which describes the propagation of waves produced by unit impulse given at position $y=\left(0, y_{2}, \cdots, y_{n}\right)$ in $\mathbb{R}_{+}^{n}$. Write

$$
\begin{aligned}
& G(x, y)=E(x-y)-F(x, y), \\
& x \in \mathbb{R}_{+}^{n}, \quad x_{1}>0, \quad y=\left(0, y_{2}^{\prime}, \cdots, y_{n}\right) \in \mathbb{R}_{+}^{n},
\end{aligned}
$$

where $E(x)$ is the fundamental solution of the Cauchy problem represented by

$$
E(x)=(2 \pi)^{-n} \int_{\boldsymbol{R}^{n-i \eta}} \exp [i x \cdot \xi] P(\xi)^{-1} d \xi, \quad \eta \in \gamma_{0} \vartheta+\Gamma .
$$

Then $F(x, y)$ is written in the form

$$
\begin{aligned}
F(x, y)=(2 \pi)^{-(n+1)} & \int_{R^{n+1}-i r \tilde{\vartheta}} i^{-1} \sum_{j, k=1}^{l} \exp \left[i \left\{\left(x^{\prime}-y^{\prime}\right) \cdot \xi^{\prime}\right.\right. \\
& \left.\left.+x_{n} \xi_{n}-y_{n} \hat{\xi}_{n+1}\right\}\right] R_{j k}\left(\xi^{\prime}\right) B_{k}\left(\xi^{\prime}, \xi_{n+1}\right) \\
& \times \xi_{n}^{j-1}\left(R\left(\xi^{\prime}\right) P_{+}(\xi) P\left(\hat{\xi}^{\prime}, \xi_{n+1}\right)\right)^{-1} d \tilde{\xi}
\end{aligned}
$$

where $r>r_{1}, \tilde{\vartheta}=(\vartheta, 0) \in \mathbb{R}^{n+1}$ and $R_{j k}\left(\xi^{\prime}\right)=(k, j)$-cofactor of $L\left(\xi^{\prime}\right)$ (see $[3],[4],[6]) . F(x, y)$ has to be interpreted in the sense of distribution with respect to $(x, y)$ in $\boldsymbol{R}_{+}^{n} \times \boldsymbol{R}_{+}^{n}$. We put

$$
\widetilde{F}(\widetilde{z})=F\left(z^{\prime}, z_{n}, 0,-z_{n+1}\right), \quad \widetilde{z}=\left(z, z_{n+1}\right) \in X=\mathbb{R}^{n-1} \times \mathbb{R}_{+}^{1} \times \mathbb{R}_{-}^{1},
$$

where $\mathbb{R}_{-}^{1}=\{\lambda \in \mathbb{R} ; \lambda<0\}$, and regard $\widetilde{F}(\widetilde{z})$ as a distribution on $X$. We note that $\widetilde{F}(\widetilde{z})$ can be regarded as a distribution on $\mathbb{R}^{n-1}$ and that supp $\widetilde{F}$ $\subset\left\{\widetilde{z} \in \boldsymbol{R}^{n+1} ; z_{n} \geq 0\right\}$. In order to investigate the wave front set $W F(G)$ of $G(x, y)$ it suffices to study $W F(\widetilde{F})$. Our main result is stated as follows:

Theorem 1.1. Assume that the condition $(A)$ is satisfied and that $\tilde{\xi}^{0} \in \mathbb{R}^{n+1}$. Then we have

$$
\begin{aligned}
& t^{V / L}\left\{t^{p_{0}} \exp \left[-i t \widetilde{\mathcal{z}} \cdot \widetilde{\xi}^{0}\right] \widetilde{F}(\widetilde{z})-\sum_{j=0}^{N} \widetilde{F}_{\tilde{\xi}_{0}, j}(\widetilde{z}) t^{-j / L}\right\} \rightarrow 0 \\
& \text { as } t \rightarrow \infty \text {, in } \mathscr{D}^{\prime}(X), N=0,1,2, \cdots,
\end{aligned}
$$

where $p_{0}$ is a rational number and $L$ is a positive integer. Moreover we have 


$$
\begin{aligned}
& \bigcup_{\tilde{\xi} \in \boldsymbol{R}^{n+1} \backslash\{0\}} \bigcup_{j=0}^{\infty} \operatorname{supp} \widetilde{F}_{\tilde{\xi}, j}(\widetilde{z}) \times\{\tilde{\xi}\} \subset W F(\widetilde{F}(\widetilde{z})) \\
& \subset W F_{A}(\widetilde{F}(\widetilde{z})) \subset \bigcup_{\tilde{\xi} \in \boldsymbol{R}^{n+1} \backslash\{0\}} K_{\tilde{\xi}}^{0} \times\{\tilde{\xi}\},
\end{aligned}
$$

$$
\overline{\operatorname{ch}}^{\dagger}\left[\bigcup_{j=0}^{\infty} \operatorname{supp} \widetilde{F}_{\tilde{\xi} 0, j}(\widetilde{z})\right] \subset K_{\tilde{\xi} 0}
$$

$$
K_{\tilde{\xi}_{0}} \subset K_{\tilde{\xi}^{0}}^{0}
$$

where

$$
\begin{aligned}
& K_{\tilde{\xi}^{0}}=\left\{\widetilde{z} \in X ; \widetilde{z} \cdot \tilde{\eta} \geq 0 \text { for all } \tilde{\eta} \in \Gamma_{\tilde{\xi}^{0}}\right\}, \\
& K_{\tilde{\xi}^{0}}^{0}=\left\{\widetilde{z} \in X ; \widetilde{z} \cdot \tilde{\eta} \geq 0 \text { for all } \tilde{\eta} \in \Gamma_{\tilde{\xi}^{0}}^{0}\right\}
\end{aligned}
$$

and $\Gamma_{\tilde{\tilde{\xi}_{0}} 0}$ and $\Gamma_{\tilde{\xi}^{0}}^{0}$ are defined by (3.3) and (3.4), respectively.

Remark. The inclusion of (1.1) can be replaced by the equality except in certain exceptional cases (see Example 5.1 in [8]).

The remainder of this paper is organized as follows. In Section 2 we shall study some properties of symmetric functions of $\lambda_{1}^{+}\left(\xi^{\prime}\right), \cdots, \lambda_{l}^{+}\left(\xi^{\prime}\right)$. In Section 3 Theorem 1.1 will be proved. In Section 4 we shall give some remarks and examples.

\section{$\S$ 2. Algebraic Considerations}

In this section we assume without loss of generality that $P(\xi)$ is irreducible. Let $\xi^{0 \prime}$ be fixed in $\mathbb{R}^{n-1}$ and $m_{\xi_{0}}^{\prime}$ the multiplicity of $\xi^{0 \prime}$ relative to $P_{m}\left(\xi^{\prime}\right)$. Let $\xi_{n}^{0} \in \mathbb{R}$ and write

$$
\nu^{m} P\left(\nu^{-1} \xi^{0}+\eta\right)=\sum_{j=m}^{m} \nu_{\xi^{0}}^{j} Q_{\xi^{0}, j}(\eta), Q_{\xi^{0}, m_{\xi}^{0}}(\eta) \not \equiv 0
$$

It is easy to see that $Q_{\xi^{0}, m_{\xi^{0}}}(\eta)=P_{\xi^{0}}(\eta)$,

$$
Q_{\xi^{0}, j}(\eta)=\sum_{|\alpha|+k=j} \frac{1}{\alpha !} \partial^{|\alpha|} / \partial \xi^{\alpha} P^{k}\left(\xi^{0}\right) \cdot \eta^{\alpha},
$$

where $P(\xi)=P^{0}(\xi)+P^{1}(\xi)+\cdots+P^{m}(\xi)$ and $P^{k}(\xi)$ is a homogeneous

\footnotetext{
$+\overline{\operatorname{ch}}[M]$ denotes the closed convex hull of $M$ in $X$.
} 
polynomial of degree $m-k$. We can write

$$
Q_{\hat{\xi}^{0}, j}(\eta)=\sum_{k=0}^{r_{j}} q_{\xi^{0}, j k}\left(\eta^{\prime}\right) \eta_{n}^{k}, \quad q_{\hat{\xi}^{0}, j r_{j}}\left(\eta^{\prime}\right) \not \equiv 0 \quad \text { if } Q_{\xi^{0}, j}(\eta) \not \equiv 0,
$$

where $r_{j} \equiv r_{j}\left(\xi^{0}\right)$ depends on $\xi^{0}$. It follows that $r_{m^{\prime}+m_{\xi^{0}}^{\prime}}=m^{\prime}$ and $r_{j}<m^{\prime}$ if $j<m^{\prime}+m_{\xi 0 \%}^{\prime}$. We put

$$
\begin{aligned}
& j_{1} \equiv j_{1}\left(\xi^{0}\right)=m^{\prime}+m_{\xi^{0}}^{\prime}, \\
& l_{k} \equiv l_{k}\left(\xi^{0}\right)=\min \left\{\left(r_{j_{k}}-r_{j}\right) /\left(j_{k}-j\right) ; m_{\xi^{0}} \leq j<j_{k}\right\}, \\
& j_{k+1} \equiv j_{k+1}\left(\xi^{0}\right)=\min \left\{j ; m_{\xi^{0}} \leq j<j_{k} \text { and }\left(r_{j_{k}}-r_{j}\right) /\left(j_{k}-j\right)=l_{k}\right\},
\end{aligned}
$$

and obtain the sequence $\left\{j_{k}, l_{k}\right\}_{k=0, \ldots, s+1}$ so that

$$
\begin{aligned}
& j_{0}=m>j_{1}=m^{\prime}+m_{\xi^{\prime}}^{\prime}>j_{2}>\cdots>j_{s}>j_{s+1}=m_{\xi^{0}}, \\
& l_{0}=0<l_{1}<l_{2}<\cdots<l_{s}<l_{s+1}=\infty,
\end{aligned}
$$

where $s \equiv s\left(\xi^{0}\right)$ depends on $\xi^{0}$. For $\rho>0$ we define the modified localization $P_{\rho, \xi 0}(\eta ; \lambda)$ of $P$ at $\xi^{0}$ by

$$
\begin{gathered}
\nu^{m} P\left(\nu^{-1} \xi^{0 \prime}+\eta^{\prime}, \nu^{-1} \xi_{n}^{0}+\nu^{-1 / \rho} \lambda+\eta_{n}\right)=\nu^{m} \xi^{0(\rho)}\left(P_{\rho, \xi^{0}}(\eta ; \lambda)+o(1)\right) \\
\text { as } \nu \downarrow 0, P_{\rho, \xi^{0}}(\eta ; \lambda) \neq 0 \text { in }(\eta, \lambda) .
\end{gathered}
$$

Then we have

$$
\begin{aligned}
& P_{\rho, \xi^{0}}(\eta ; \lambda)=q_{\xi^{0}, j_{k} r} j_{j_{k}}\left(\eta^{\prime}\right) \lambda^{r_{j_{k}}}, \\
& m_{\xi^{0}}(\rho)=j_{k}-r_{j_{k}} / \rho,
\end{aligned}
$$

if $l_{k}>\rho>l_{k-1}, 1 \leq k \leq s+1$, and we have

$$
\begin{gathered}
P_{\rho, \xi^{0}}(\eta ; \lambda)=\left[q_{\xi^{0}, j_{k}{ }^{r} j_{k}}\left(\eta^{\prime}\right) \lambda^{r} j_{j_{k}}^{-r} j_{j_{k+1}}\right. \\
\left.+\cdots+q_{\xi^{0}, j_{k+1}{ }^{r} j_{k+1}}\left(\eta^{\prime}\right)\right] \lambda^{r} j_{k+1}, \\
m_{\xi^{0}}(\rho)=j_{k}-r_{j_{k}} / \rho=j_{k+1}-r_{j_{k+1}} / \rho,
\end{gathered}
$$

if $\rho=l_{k}, 1 \leq k \leq s$. Moreover we have

$$
P_{\rho, \xi^{0}}(\eta ; \lambda)=P_{\xi^{0}}\left(\eta^{\prime}, \lambda+\eta_{n}\right), \quad m_{\xi^{0}}(\rho)=m_{\xi^{0}},
$$

if $\rho=l_{s+1}=\infty$. We note that $j_{k}\left(\xi^{0}\right)$ and $l_{k-1}\left(\xi^{0}\right)$ are independent of $\xi_{n}^{0}$ if $l_{k-1}<1$. In fact, we have

$$
P_{\rho, \xi^{0}}(\eta ; \lambda)=P_{\rho,\left(\xi^{\circ}, 0\right)}(\eta ; \lambda) \quad \text { if } \quad l_{k-1}<\rho<\min \left(1, l_{k}\right) .
$$


Now we define the modified principal part $p_{\rho}^{0}(\eta ; \lambda)$ and modified degree $\operatorname{deg}_{\rho} p=\sigma$ for a polynomial $p(\eta ; \lambda)$ by

$$
\begin{aligned}
& p\left(t \eta ; t^{(\rho-1) / \rho} \lambda\right)=t^{\sigma}\left(p_{\rho}^{0}(\eta ; \lambda)+o(1)\right) \text { as } t \rightarrow \infty, \\
& p_{\rho}^{0}(\eta ; \lambda) \not \equiv 0 \text { in }(\eta, \lambda) .
\end{aligned}
$$

Lemma 2. 1. Let $\rho>0$ and put $P_{\rho, \xi^{0}}^{0}(\eta ; \lambda)=\left(P_{\rho, \xi^{0}}\right)_{\rho}^{0}(\eta ; \lambda)$. Then we have

$$
P_{\rho, \xi^{0}}^{0}(\eta ; \lambda)=\left(P^{0}\right)_{\rho, \xi^{0}}(\eta ; \lambda), \quad \operatorname{deg}_{\rho} P_{\rho, \xi^{0}}=m_{\xi^{0}}(\rho)
$$

Proof.

$$
\begin{aligned}
\nu^{m} P^{0}\left(\nu^{-1} \xi^{0 \prime}\right. & \left.+\eta^{\prime}, \nu^{-1} \xi_{n}^{0}+\nu^{-1 / \rho} \lambda+\eta_{n}\right) \\
& =\nu^{\sigma_{0}}\left(\left(P^{0}\right)_{\rho, \xi^{0}}(\eta ; \lambda)+Q(\eta, \lambda ; \nu)\right),
\end{aligned}
$$

where $Q(\eta, \lambda ; \nu)$ is a polynomial in $(\eta, \lambda)$, continuous in $(\eta, \lambda, \nu)$ and $Q(\eta, \lambda ; 0)=0$. Therefore we have

$$
\begin{aligned}
& \nu^{m} \partial^{|\alpha|} / \partial \eta^{\alpha} P^{0}\left(\nu^{-1} \xi^{0 \prime}+\eta^{\prime}, \nu^{-1} \xi_{n}^{0}+\nu^{-1 / \rho} \lambda+\eta_{n}\right) \\
& \quad=\nu^{\sigma_{0}}\left(\partial^{|\alpha|} / \partial \eta^{\alpha}\left(P^{0}\right)_{\rho, \xi^{0}}(\eta ; \lambda)+\partial^{|\alpha|} / \partial \eta^{\alpha} Q(\eta, \lambda ; \nu)\right) .
\end{aligned}
$$

From this it follows that

$$
\begin{aligned}
\nu^{m} \widetilde{P}^{0} & \left(\nu^{-1} \xi^{0 \prime}+\eta^{\prime}, \nu^{-1} \xi_{n}^{0}+\nu^{-1 / \rho} \lambda+\eta_{n}\right) \\
& =\nu^{\sigma_{0}}\left(\left(\left(P^{0}\right)_{\rho, \xi_{0}}\right)^{\sim}(\eta ; \lambda)^{2}+0(1)\right)^{1 / 2} \text { as } \nu \downarrow 0,
\end{aligned}
$$

where $\widetilde{p}(\eta ; \lambda)^{2}=\sum\left|\partial^{|\alpha|} / \partial \eta^{\alpha} p(\eta ; \lambda)\right|^{2}$. Hyperbolicity of $P$ implies that

$$
\begin{aligned}
& \left|P\left(\nu^{-1} \xi^{0 \prime}+\eta^{\prime}, \nu^{-1} \xi_{n}^{0}+\nu^{-1 / \rho} \lambda+\eta_{n}\right)\right| \\
& \quad \leq \text { const. } \times \widetilde{P}^{0}\left(\nu^{-1} \xi^{0 \prime}+\eta^{\prime}, \nu^{-1} \xi_{n}^{0}+\nu^{-1 / \rho} \lambda+\eta_{n}\right), \lambda \in \boldsymbol{R}, \eta \in \boldsymbol{R}^{n}
\end{aligned}
$$

(see [5]). Since there exists $\left(\eta^{0}, \lambda_{0}\right) \in \boldsymbol{R}^{n+1}$ such that $P_{\rho, \xi^{0}}\left(\eta^{0} ; \lambda_{0}\right) \neq 0$, it follows that $\sigma_{0} \leq m_{\xi^{0}}(\rho)$. Put

$$
\begin{aligned}
\nu^{m} P^{k} & \left(\nu^{-1} \xi^{0 \prime}+\eta^{\prime}, \nu^{-1} \xi_{n}^{0}+\nu^{-1 / \rho} \lambda+\eta_{n}\right) \\
& =\nu^{\sigma_{k}}\left(\left(P^{k}\right)_{\rho, \xi^{0}}(\eta ; \lambda)+o(1)\right) \text { as } \nu \downarrow 0 .
\end{aligned}
$$

Then we have $\operatorname{deg}_{\rho}\left(P^{k}\right)_{\rho, \xi^{0}}=\sigma_{k}-k$ and $\left(P^{k}\right)_{\rho, \xi^{0}}^{0}=\left(P^{k}\right)_{\rho, \xi^{0}}$. Therefore it follows that $\sigma_{0}=m_{\xi^{0}}(\rho)$. This proves the lemma. Q.E.D. 
Lemma 2. 2. Let $\rho>0, \rho \neq 1$ and $\lambda_{0} \in \mathbb{R} \backslash\{0\}$. Then $P_{\rho, \xi^{0}}\left(\eta ; \lambda_{0}\right)$ is a hyperbolic polynomial with respect to $\vartheta$. Moreover we have

(2.3) $P_{\rho, \xi^{0}}\left(\eta ; \lambda_{0}\right) \neq 0 \quad$ for $\eta \in\left\{\begin{array}{l}\mathbb{R}^{n}-i \gamma_{0} \vartheta-i \Gamma\left(\left(P_{(0,1)}\right)_{\xi^{0}}\right) \text { if } 1>0>0, \\ \mathbb{R}^{n}-i \gamma_{0} \vartheta-i \Gamma\left(\left(P_{\xi^{0}}\right)_{(0,1)}\right) \text { if } \infty>0>1, \\ \mathbb{R}^{n}-i \gamma_{0} \vartheta-i \Gamma_{\xi^{0}} \text { if } \rho=l_{s+1}=\infty .\end{array}\right.$

In particular,

$$
\Gamma\left(P_{\rho, \xi^{0}}\left(\eta ; \lambda_{0}\right)\right) \supset\left\{\begin{array}{l}
\Gamma\left(\left(P_{(0,1)}\right)_{\xi_{0} 0}\right) \text { if } 1>\rho>0, \\
\Gamma\left(\left(P_{\xi^{0}}\right)_{(0,1)}\right) \text { if } \infty>\rho>1, \\
\Gamma_{\xi^{0}} \text { if } \rho=l_{s+1}=\infty,
\end{array}\right.
$$

and

$$
\left(P_{\rho, \xi^{0}}\right)^{0}\left(\eta ; \lambda_{0}\right)=\left\{\begin{array}{l}
\left(P_{(0,1)}^{0}\right)_{\xi^{0}}(\eta) \lambda_{0}^{m^{\prime}} \text { if } l_{1} \geq \rho>0, \\
\left(P_{\xi^{0}}^{0}\right)_{(0,1)}(\eta) \lambda_{0}^{r_{s+1}} \text { if } \infty>\rho \geq l_{s} \\
P_{\xi^{0}}^{0}(\eta) \text { if } \rho=l_{s+1}=\infty,
\end{array}\right.
$$

where $\left(P_{\rho, \xi^{0}}\right)^{0}\left(\eta ; \lambda_{0}\right)$ denotes the principal part of a polynomial $P_{\rho, \xi^{0}}\left(\eta ; \lambda_{0}\right)$ in $\eta$.

Remark. We note that $\Gamma_{\xi^{0}} \subset \Gamma\left(\left(P_{\xi^{0}}\right)_{(0,1)}\right)$ and that $\left(P_{(0,1)}\right)_{\xi^{0}}(\eta)$ is independent of $\xi_{n}^{0}$.

Proof. Since $\rho \neq 1$, it follows that $P_{\rho, \xi^{0}}\left(\eta ; \lambda_{0}\right) \not \equiv 0$ in $\eta$. In fact, from Lemma 2.1 we have

$$
\operatorname{deg} q_{\xi^{0}, j_{k} r_{j_{k}}}\left(\eta^{\prime}\right)=j_{k}-r_{j_{k}}
$$

Thus

$$
\left(P_{\rho, \xi_{0} 0}\right)^{0}\left(\eta ; \lambda_{0}\right)=\left\{\begin{aligned}
&\left(q_{\xi^{0}, j_{k} r_{j_{k}}}\right)^{0}\left(\eta^{\prime}\right) \lambda_{0}^{r_{j_{k}}} \text { if } \quad l_{k} \geq \rho>l_{k-1} \\
& \text { and } 1>\rho>0, \\
&\left(q_{\xi^{0}, j_{k} r_{j_{k}}}\right)^{0}\left(\eta^{\prime}\right) \lambda_{0}^{r_{j_{k}}} \text { if } \quad l_{k}>\rho \geq l_{k-1} \\
& \text { and } \rho>1, \\
& P_{\xi^{0}}^{0}(\eta) \text { if } \rho=l_{s+1}=\infty .
\end{aligned}\right.
$$

Now let us assume that there exists $\eta^{0} \in \mathbb{R}^{n}-i \gamma_{0} \vartheta-i \Gamma$ such that $P_{\rho, \xi^{0}}\left(\eta^{0} ; \lambda_{0}\right)=0$. Then there exist positive numbers $\varepsilon, \delta$ and $\zeta^{0} \in \mathbb{C}^{n}$ such that

$$
\begin{aligned}
& \left|P_{\rho, \xi^{0}}\left(\eta^{0}+\mu \zeta^{0} ; \lambda_{0}\right)\right|>\varepsilon>0 \quad \text { for } \quad|\mu|=\delta>0, \\
& \eta^{0}+\mu \zeta^{0} \in \boldsymbol{R}^{n}-i \gamma_{0} \vartheta-i \Gamma \quad \text { for } \quad|\mu| \leq \delta .
\end{aligned}
$$


Therefore from Rouchés theorem it follows that there exists a positive number $\nu_{0}$ such that $P\left(\nu^{-1} \xi^{0 \prime}+\eta^{0 \prime}+\mu \zeta^{0 \prime}, \nu^{-1} \xi_{n}^{0}+\nu^{-1 / \rho} \lambda_{0}+\eta_{n}^{0}+\mu \zeta_{n}^{0}\right)$ has zeros within $|\mu|<\delta$ if $0<\nu \leq \nu_{0}$, which is a contradiction to $P(\xi) \neq 0$ for $\xi \in \mathbb{R}^{n}$ $-i \gamma_{0} \vartheta-i \Gamma$. So we have

$$
P_{\rho, \xi^{0}}\left(\eta ; \lambda_{0}\right) \neq 0 \quad \text { for } \eta \in \mathbb{R}^{n}-i \gamma_{0} \vartheta-i \Gamma \text {. }
$$

This implies that $P_{\rho, \xi^{0}}\left(\eta ; \lambda_{0}\right)$ is a hyperbolic polynomial with respect to $\vartheta$ and that $\Gamma\left(P_{\rho, \xi^{\circ}}\left(\eta ; \lambda_{0}\right)\right) \supset \Gamma$. Next let us prove (2.4). We note that (2.3) follows from (2.4) (see [1], [3]). One can easily verify (2.5). Therefore (2.4) holds when $\infty \geq \rho \geq l_{s}$ or $l_{1} \geq \rho>0$. Let us prove (2.4) when $1>\rho>0$. For we can prove (2.4) in the same manner when $\rho>1$. Now assume that $\Gamma\left(P_{\rho, \xi^{0}}\left(\eta ; \lambda_{0}\right)\right) \supset \Gamma\left(\left(P_{(0,1)}\right)_{\xi^{0}}\right)$ when $1>l_{k}>\rho>0$. Then by (2.1) we have

$$
\Gamma\left(q_{\xi^{0}, j_{k} r_{j_{k}}}\left(\eta^{\prime}\right)\right) \supset \Gamma\left(\left(P_{(0,1)}\right)_{\xi^{0}}\right) .
$$

Thus from (2.6) it follows that

$$
P_{l_{k}, \xi^{0}}\left(\eta ; \lambda_{0}\right) \neq 0 \quad \text { for } \eta \in \mathbb{R}^{n}-i \gamma_{0} \vartheta-i \Gamma\left(\left(P_{(0,1)}\right)_{\xi^{0}}\right) \text {. }
$$

Assume that

$$
q_{\xi^{0}, j_{k+1} r_{j_{k+1}}}\left(\eta^{0 \prime}\right)=0 \quad \text { for some } \eta^{0} \in \mathbb{R}^{n}-i \gamma_{0} \vartheta-i \Gamma\left(\left(P_{(0,1)}\right)_{\xi^{0}}\right) .
$$

From (2.2) we have

$$
\lambda^{-r_{j_{k+1}}} P_{l_{k}, \hat{\xi}^{0}}(\eta ; \lambda) \rightarrow q_{\xi^{0}, j_{k+1}} r_{j_{k+1}}\left(\eta^{\prime}\right) \text { as } \lambda \downarrow 0
$$

(locally uniform), which leads us to a contradiction, using Rouché's theorem. Therefore,

$$
P_{\rho, \xi^{0}}\left(\eta ; \lambda_{0}\right)=q_{\xi^{0}, j_{k+1} r_{j_{k+1}}}\left(\eta^{\prime}\right) \lambda_{0}^{r_{j_{k+1}}} \neq 0
$$

when $l_{k+1}>\rho>l_{k}$ and $\eta \in \mathbb{R}^{n}-i \gamma_{0} \vartheta-i \Gamma\left(\left(P_{(0,1)}\right)_{\xi^{0}}\right)$. From (2.7) and (2.8) it follows that

$$
\Gamma\left(P_{\rho, \xi^{0}}\left(\eta ; \lambda_{0}\right)\right) \supset \Gamma\left(\left(P_{(0,1)}\right)_{\xi^{0}}\right) \quad \text { when } \quad l_{k+1}>\rho>0 .
$$

Q.E.D.

We define $q \equiv q\left(\xi^{\prime \prime}\right)$ by

(2. 9) $\partial^{k} / \partial \xi_{1}^{k} P^{0}\left(\xi^{0 \prime}, \lambda\right) \equiv 0, \quad 0 \leq k \leq q-1, \quad \partial^{q} / \partial \xi_{1}^{q} P^{0}\left(\xi^{0 \prime}, \lambda\right) \not \equiv 0$ in $\lambda$.

Put 


$$
p \equiv p\left(\xi^{0 \prime}\right)=\operatorname{deg} \partial^{q} / \partial \xi_{1}^{q} P^{0}\left(\xi^{0 \prime}, \lambda\right),
$$

and define $r \equiv r\left(\xi^{0}\right)$ by

$$
\begin{aligned}
& \partial^{q+k} / \partial \xi_{1}^{q} \partial \xi_{n}^{k} P^{0}\left(\xi^{0}\right)=0, \quad 0 \leq k \leq r-1, \\
& \partial^{q+r} / \partial \xi_{1}^{a} \partial \xi_{n}^{r} P^{0}\left(\xi^{0}\right) \neq 0 .
\end{aligned}
$$

Then we have the following

\section{Lemma 2.3.}

$$
\begin{aligned}
& q \leq m_{\xi^{0}}^{\prime} \leq m-m^{\prime}, \quad p \leq \min \left(m^{\prime}, m-q\right), \\
& q \leq m_{\xi^{0}} \leq q+r \leq q+p \leq m^{\prime}+m_{\xi^{0}}^{\prime} \leq m, \\
& p=m^{\prime}, \quad m_{\xi^{0}}^{\prime}=m-m^{\prime} \quad \text { if } q=m-m^{\prime} .
\end{aligned}
$$

Moreover

$$
r_{j} \leq j-q \text { for } m_{\xi^{0}} \leq j \leq m,
$$

$$
r_{j}<j-q \text { if } m_{\xi^{0}} \leq j<q+r \text { or } q+p<j \leq m \text {, }
$$

$$
r_{j}<m^{\prime} \quad \text { if } m_{\xi^{0}} \leq j<m^{\prime}+m_{\xi^{\prime}}^{\prime} \text {, }
$$

$$
r_{q+r}=r, \quad r_{q+p}=p \quad \text { and } \quad r_{m^{\prime}+m_{\xi^{\prime}}^{\prime}}=m^{\prime}
$$

Remark. This lemma yields us the following Newton polygon (Fig. 1).

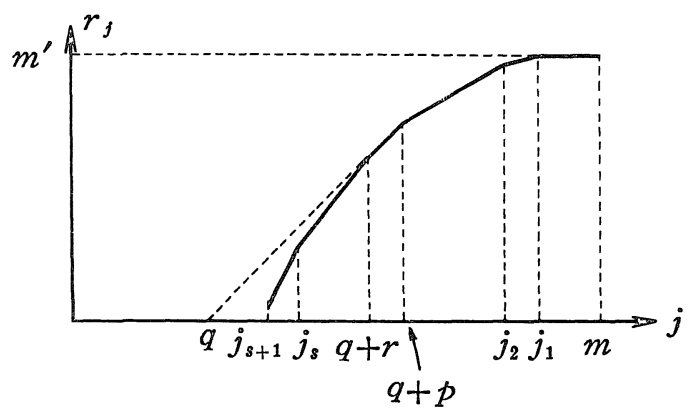

Fig. 1.

Proof. If $|\alpha|+k<q$,

$$
\partial^{|\alpha|} / \partial \xi^{\alpha} P^{k}\left(\xi^{0 \prime}, \lambda\right) \equiv 0 \quad \text { in } \lambda .
$$

In fact, for each $\lambda_{0} \in \mathbb{R}$ 


$$
\begin{aligned}
& \nu^{m} P\left(\nu^{-1} \xi^{0 \prime}+\eta^{\prime}, \nu^{-1} \lambda_{0}+\eta_{n}\right) \\
& \quad=\sum_{j=0}^{m} \nu^{j} \sum_{|\alpha|+k=j} \frac{1}{\alpha !} \partial^{|\alpha|} / \partial \xi^{\alpha} P^{k}\left(\xi^{0 \prime}, \lambda_{0}\right) \eta^{\alpha} .
\end{aligned}
$$

If $\partial^{|\alpha|} / \partial \xi^{\alpha} P^{k}\left(\xi^{\prime \prime}, \lambda_{0}\right) \neq 0$ for some $\alpha$ and $k$ with $|\alpha|+k<q$, hyperbolicity of $P$ implies that there exists a non-negative integer $h$ such that $h \leq|\alpha|$ $+k<q$ and $\partial^{h} / \partial \xi_{1}^{h} P^{0}\left(\xi^{0 \prime}, \lambda_{0}\right)=h ! P_{\left(\xi^{\circ}, \lambda_{0}\right)}^{0}(\vartheta) \neq 0$, which is a contradiction to (2.9). (2.13) easily follows from (2.17). (2.12), (2.15) and (2.16) are obvious. Now assume that

$$
p^{\prime} \equiv \max \left\{\operatorname{deg} \partial^{\left|\alpha^{\prime}\right|} / \partial \xi^{\prime \alpha^{\prime}} P^{k}\left(\xi^{0 \prime}, \lambda\right) ;\left|\alpha^{\prime}\right|+k=q\right\}>p .
$$

Then we have

$$
P_{\rho,(\xi 0,0)}^{0}(\vartheta ; \lambda)=0 \quad \text { for } 1>\rho>\left(m^{\prime}-p\right) /\left(m^{\prime}+1-p\right),
$$

which is a contradiction to hyperbolicity of $P_{\rho,\left(\xi^{0}, 0\right)}\left(\eta ; \lambda_{0}\right), \lambda_{0} \in \mathbb{R} \backslash\{0\}$. In fact, we have $r_{q+p^{\prime}}=p^{\prime}$ and $r_{j}<j-q$ for $q+p^{\prime}<j \leq m$. Therefore, $j-r_{j} / \rho$ $>q+p^{\prime}-p^{\prime} / \rho$ when $1>\rho>\left(m^{\prime}-p^{\prime}\right) /\left(m^{\prime}+1-p^{\prime}\right)$ and $j \neq q+p^{\prime}$. For it is obvious that $j-r_{j} / \rho \geq j(1-1 / \rho)+q / \rho>q+p^{\prime}-p^{\prime} / \rho$ if $j<q+p^{\prime}$. If $j>q+p^{\prime}$, then

$$
j-r_{j} / \rho=j-r_{j}+(1-1 / \rho) r_{j} \geq q+1+(1-1 / \rho) m^{\prime}>q+p^{\prime}-p^{\prime} / \rho .
$$

Thus we have $P_{\rho,\left(\xi^{\prime}, 0\right)}(\eta ; \lambda)=q_{\left(\xi^{\prime} 0^{\prime}, 0\right), q^{+} p^{\prime} p^{\prime}}\left(\eta^{\prime}\right) \lambda^{p^{\prime}}$. Since $q_{\left(\xi^{\prime}, 0\right), q+p^{\prime} p^{\prime}}^{0}\left(\vartheta^{\prime}\right)$ $=\left(q ! p^{\prime} !\right)^{-1} \partial^{q+p^{\prime}} / \partial \xi_{1}^{q} \partial \xi_{n}^{p^{\prime}} P^{0}\left(\xi^{\prime \prime}, 0\right)$ we obtain (2.18). Therefore we have

$$
p=\max \left\{\operatorname{deg} \partial^{\left|\alpha^{\prime}\right|} / \partial \xi^{\prime \alpha^{\prime}} P^{k}\left(\xi^{\prime \prime}, \lambda\right) ;\left|\alpha^{\prime}\right|+k=q\right\} .
$$

This implies that $r_{j}<j-q$ if $q+p<j \leq m$. Next let us prove that

$$
\partial^{\left|\alpha^{\prime}\right|+h} / \partial \xi^{\prime \alpha^{\prime}} \partial \xi_{n}^{h} P^{k}\left(\xi^{0}\right)=0 \text { for }\left|\alpha^{\prime}\right|+k=q \text { and } 0 \leq h \leq r-1 .
$$

Assume that

$$
\begin{array}{r}
r^{\prime}=\min \left\{h ; \partial^{\left|\alpha^{\prime}\right|+h} / \partial \xi^{\alpha^{\prime}} \partial \xi_{n}^{h} P^{k}\left(\xi^{0}\right) \neq 0 \text { for some } \alpha^{\prime}\right. \\
\text { and } \left.k \text { with }\left|\alpha^{\prime}\right|+k=q\right\}<r .
\end{array}
$$

Then similarly we have

$$
P_{\rho, \xi^{0}}^{0}(\vartheta ; \lambda)=0 \quad \text { for }\left(q+r^{\prime}-m_{\xi^{0}}+1\right) /\left(q+r^{\prime}-m_{\xi^{0}}\right)>0>1,
$$

which is a contradiction to hyperbolicity of $P_{\rho, \xi^{0}}\left(\eta ; \lambda_{0}\right), \lambda_{0} \in \boldsymbol{R} \backslash\{0\}$. From (2.19) it follows that $r_{j}<j-q$ if $m_{\xi^{0}} \leq j<q+r_{\text {。 }}$ 
From Lemma 2.3 it follows that there exist positive integers $t$ $\equiv t\left(\xi^{\prime \prime}\right)$ and $t^{\prime} \equiv t^{\prime}\left(\xi^{0}\right)$ such that $1 \leq t \leq t^{\prime} \leq s+1, j_{t}=q+p$ and $j_{t^{\prime}}=q+r$. If $r<p$, then $t^{\prime}=t+1$ and $l_{t}=1$. If $r=p$, then $t=t^{\prime}, l_{t}>1$ and $l_{t-1}<1$. Thus $j_{k}\left(\xi^{0}\right)$ and $l_{k-1}\left(\xi^{0}\right), 0 \leq k \leq t\left(\xi^{0 \prime}\right)$, are independent of $\xi_{n}^{0}$. Put

$$
\begin{aligned}
& P_{l_{k}, \xi^{0}}(\eta ; \lambda)=P_{k, \xi^{0}}\left(\eta^{\prime} ; \lambda\right) \lambda^{r_{j_{k+1}}}, \\
& P_{k, \xi^{0}}\left(\eta^{\prime} ; \lambda\right)=q_{\xi^{0}, j_{k} r_{j_{k}}}\left(\eta^{\prime}\right) \lambda^{r_{j_{k}}-r_{j_{k+1}}}+\cdots+q_{\xi^{0}, j_{k+1} r_{j_{k+1}}}\left(\eta^{\prime}\right) .
\end{aligned}
$$

By Lemma 2.2 we obtain the following

Lemma 2. 4. For $1 \leq k<t P_{k, \xi^{0}}\left(\eta^{\prime} ; \lambda\right)$ has no real zeros when $\eta^{\prime}$ $\in \mathbb{R}^{n-1}-i \gamma_{0} \vartheta^{\prime}-i \dot{\Gamma}\left(\left(P_{(0,1)}\right)_{\xi^{0}}\right)$. For $t^{\prime} \leq k \leq s P_{k, \xi^{0}}\left(\eta^{\prime} ; \lambda\right)$ has no real zeros when $\eta^{\prime} \in \mathbb{R}^{n-1}-i \gamma_{0} \vartheta^{\prime}-i \dot{\Gamma}\left(\left(P_{\xi_{0}}\right)_{(0,1)}\right)$.

Denote the roots of $P_{\xi^{0}}\left(\eta^{\prime}, \lambda\right)=0$ by $\lambda_{\xi^{0}, 1}^{+}\left(\eta^{\prime}\right), \cdots, \lambda_{\xi^{0}, l^{\prime}}^{+}\left(\eta^{\prime}\right), \lambda_{\xi_{0}, 1}^{-}\left(\eta^{\prime}\right), \cdots$, $\lambda_{\xi^{-0}, r_{\xi_{\xi}^{0}-l^{\prime}}}\left(\eta^{\prime}\right)$ so that the $\lambda_{\xi_{\xi^{0}}^{ \pm}, j}^{+}\left(\eta^{\prime}\right)$ are continuous and that

$\operatorname{Im} \lambda_{\xi^{0}, j}^{ \pm}\left(\eta^{\prime}-i \gamma \vartheta^{\prime}\right) \gtrless 0 \quad$ for $\gamma>\gamma_{0}$ and $\eta^{\prime} \in \mathbb{R}^{n-1}$,

when $r_{m_{\xi}} \neq 0$. Then we easily obtain the following

Lemma 2.5. Assume that $r_{m_{\xi^{0}}} \neq 0$. Then

$$
\left\{\lambda_{\dot{s}^{0}, j}^{+}\left(\eta^{\prime}\right)\right\}_{1 \leq j \leq l^{\prime}} \cap\left\{\lambda_{\xi^{0}, j}\left(\eta^{\prime}\right)\right\}_{1 \leq j \leq r_{\xi_{\xi^{0}}} l^{\prime}}=\varnothing
$$

if $\eta^{\prime} \in \mathbb{R}^{n-1}-i \gamma_{0} \vartheta^{\prime}-i \dot{\Gamma}_{\xi^{0}}$.

Put

$$
P_{\xi^{\prime \prime}, q}(\eta ; \lambda)=\sum_{|a|+k=q} \frac{1}{\alpha !} \partial^{|\alpha|} / \partial \xi^{\alpha} P^{k}\left(\hat{\xi}^{0 \prime}, \lambda\right) \eta^{\alpha}
$$

We note that $P_{\xi^{\circ}, q}(\eta ; \lambda)$ is independent of $\eta_{n}$. From the proof of Lemma 2.3 it follows that $\operatorname{deg} P_{\xi^{\circ}, q}(\eta ; \lambda) \leq p$ in $\lambda$ for fixed $\eta$. The coefficient of $\lambda^{p}$ in $P_{\xi^{\circ}, q}(\eta ; \lambda)$ is equal to $q_{\xi^{0}, q+p p}\left(\eta^{\prime}\right)$, where $\xi_{n}^{0} \in \mathbb{R}$. Since $q+p=j_{t}$, $p=r_{j_{t}}$ and $l_{t-1}<1$, it follows from (2.1) and Lemma 2.2 that

$$
q_{\xi^{0}, q+p p}\left(\eta^{\prime}\right) \neq 0 \text { for } \eta \in \mathbb{R}^{n}-i \gamma_{0}-i \Gamma\left(\left(P_{(0,1)}\right)_{\xi^{0}}\right) \text {. }
$$

Therefore we have 
$\operatorname{deg} P_{\xi^{0}, q}(\eta ; \lambda)=p$ in $\lambda$ for fixed $\eta \in \mathbb{R}^{n}-i \gamma_{0} \vartheta-i \Gamma\left(\left(P_{(0,1)}\right)_{\left(\xi^{0}, 0\right)}\right)$.

Lemma 2. 6. Let $\xi_{n}^{0} \in \boldsymbol{R}$ and $\eta \in \boldsymbol{R}^{n}-i \gamma_{0} \vartheta-i \Gamma\left(\left(P_{\xi^{0}}\right)_{(0,1)}\right)$. Then $\lambda=\xi_{n}^{0}$ is a root of $\partial^{q} / \partial \xi_{1}^{q} P^{0}\left(\xi^{0 \prime}, \lambda\right)=0$ with multiplicity $r^{\dagger}$ if and only if $\lambda=\xi_{n}^{0}$ is a root of $P_{\xi^{0}, q}(\eta ; \lambda)=0$ with multiplicity $r$.

Proof. Now assume that $P_{\xi^{0}, q}\left(\eta ; \xi_{n}^{0}\right)=0$ for some $\eta \in \mathbb{R}^{n}-i \gamma_{0} \vartheta$ $-i \Gamma\left(\left(P_{\xi^{0}}\right)_{(0,1)}\right)$. Then we have $\partial^{q} / \partial \xi_{1}^{q} P^{0}\left(\xi^{0}\right)=0$. In fact, if $\partial^{q} / \partial \xi_{1}^{q} P^{0}\left(\xi^{0}\right)$ $\neq 0$, we have $\left(P_{\xi^{0}}\right)_{(0,1)}(\xi)=P_{\xi^{0}, q}\left(\xi ; \xi_{n}^{0}\right) \neq 0$ for $\xi \in \mathbb{R}^{n}-i \gamma_{0} \vartheta-i \Gamma\left(\left(P_{\xi^{0}}\right)_{(0,1)}\right)$, which is a contradiction to $P_{\xi^{0}, q}\left(\eta ; \xi_{n}^{0}\right)=0$. Next assume that there exists a non-negative integer $k$ such that $k \leq r-1$ and $\partial^{k} / \partial \lambda^{k} P_{\xi^{\circ}, q}\left(\eta ; \xi_{n}^{0}\right)$ $\not \equiv 0$ in $\eta$. Then we have $r_{q+k}=k$, which is a contradiction to (2.14). For $l_{t}>\rho>1$ we have

$$
P_{\rho, \xi^{0}}(\eta ; \lambda)=\frac{1}{r !} \lambda^{r} \partial^{r} / \partial \lambda^{r} P_{\xi^{0 \prime}, q}\left(\eta ; \xi_{n}^{0}\right)
$$

From Lemma 2.2 and this it follows that

$$
\partial^{r} / \partial \lambda^{r} P_{\xi^{0}, q}\left(\eta ; \xi_{n}^{0}\right) \neq 0 \quad \text { for } \eta \in \boldsymbol{R}^{n}-i \gamma_{0} \theta-i \Gamma\left(\left(P_{\xi^{0}}\right)_{(0,1)}\right) .
$$

This proves the lemma.

Q.E.D.

Lemma 2.6 yields the following

Lemma 2. 7. Let $\eta \in \mathbb{R}^{n}-i \gamma_{0} \vartheta-i\left\{\underset{\xi_{n}^{0} \in \mathbb{R}}{\cap} \Gamma\left(\left(P_{\xi^{0}}\right)_{(0,1)}\right) \cap \Gamma\left(\left(P_{(0,1)}\right)_{(\xi 00,0)}\right)\right\}$. The real zeros of $\partial^{q} / \partial \xi_{1}^{q} P^{0}\left(\xi^{\prime \prime}, \lambda\right)$ agree with those of $P_{\xi^{\prime \prime}, q}(\eta ; \lambda)$ (including multiplicities). Moreover the number of the roots with positive imaginary part of $P_{\xi^{\circ}, q}(\eta ; \lambda)=0$ is equal to that of the roots with positive imaginary part of $\partial^{q} / \partial \xi_{1}^{q} P^{0}\left(\xi^{0 \prime}, \lambda\right)=0$.

Remark. The non-real zeros of $\partial^{q} / \partial \xi_{1}^{q} P^{0}\left(\xi^{0 \prime}, \lambda\right)$ do not always agree with those of $P_{\xi^{0}, q}(\eta ; \lambda)$. In fact, for $P(\xi)=P^{0}(\xi)=\xi_{1}^{4}-2 \xi_{1}^{2}\left(\xi_{2}^{2}+\xi_{3}^{2}+\xi_{4}^{2}\right)$ $+\left(\xi_{2}^{2}+\xi_{3}^{2}+\xi_{4}^{2} / 2\right) \xi_{2}^{2}$ we have

${ }^{\dagger} r=r\left(\xi^{0}\right)$ is defined by (2.10) and (2.11). Lemma 2.6 implies that $\lambda=\xi_{n}^{0}$ is a root of $P_{\xi^{0}, q}(\eta ; \lambda)=0$ with multiplicity $r$ and that $\partial^{q} / \partial \xi_{1}^{q} P^{0}\left(\xi^{0}\right)=0$ if $P_{\xi^{0}, q}\left(\eta ; \xi_{n}^{0}\right)=0$ for some $\eta \in \mathbb{R}^{n}-i \gamma_{0} \vartheta-i \Gamma\left(\left(P_{\xi^{0}}\right)(0,1)\right)$. 


$$
\begin{aligned}
& \partial^{2} / \partial \xi_{1}^{2} P^{0}(0,0,1, \lambda)=-4\left(1+\lambda^{2}\right), \\
& P_{(0,0,1), 2}(\eta ; \lambda)=-2\left(1+\lambda^{2}\right) \eta_{1}^{2}+\left(1+\lambda^{2} / 2\right) \eta_{2}^{2} .
\end{aligned}
$$

Moreover if $\partial^{q} / \partial \xi_{1}^{q} P^{0}\left(\xi^{0}\right) \neq 0$, then $P_{\xi^{0}}(\eta)=\left(P_{\xi^{0}}\right)_{(0,1)}(\eta)$ and $P_{\hat{\xi}^{0}, q}^{0}\left(-i \eta^{0} ; \xi_{n}^{0}\right)$ $=P_{\xi^{0}}^{0}\left(-i \eta^{0}\right)=0$ for $\eta^{0} \in \partial \Gamma\left(P_{\xi^{0}}\right)^{\dagger}$.

Put

$$
\begin{aligned}
& \sigma^{k}\left(\xi^{\prime}\right)=\sum_{j=1}^{l} \lambda_{j}^{+}\left(\xi^{\prime}\right)^{k}, \quad 1 \leq k \leq l^{\dagger \dagger}, \\
& \dot{\Gamma}_{\xi^{0} 0}=\bigcap_{\hat{\xi}_{n}^{0} \in \boldsymbol{R}} \dot{\Gamma}_{\xi^{0}} \cap \dot{\Gamma}\left(\left(P_{(0,1)}\right)_{\left(\xi^{0}, 0\right)}\right) .
\end{aligned}
$$

Lemma 2. 8. Let $1 \leq k \leq l$. For any compact set $K$ in $\mathbb{R}^{n-1}$ $-i \gamma_{0} \vartheta^{\prime}-i \dot{\Gamma}_{\xi^{0}}$ there exists $\nu_{K}(>0)$ such that $\sigma^{k}\left(\nu^{-1} \xi^{0 \prime}+\eta^{\prime}\right)$ is welldefined for $\eta^{\prime} \in K$ and $0<\nu \leq \nu_{K}$ and

$$
\nu^{s} \sigma^{k}\left(\nu^{-1} \xi^{0 \prime}+\eta^{\prime}\right)=\sum_{j=0}^{\infty} \sigma_{\xi^{\prime}, j}^{k}\left(\eta^{\prime}\right) \nu^{j / L}, \quad \sigma_{\xi^{\prime} 0^{\prime}, 0}^{k}\left(\eta^{\prime}\right) \not \equiv 0,
$$

whose convergence is uniform in $K \times\left\{\nu ; 0 \leq \nu \leq \nu_{K}\right\}$, where $s_{k}$ is a rational number and $L$ is a positive integer. Moreover the $\sigma_{\xi_{00}, j}^{k}$ are holomorphic in $\mathbb{R}^{n-1}-i \gamma_{0} \vartheta^{\prime}-i \dot{\Gamma}_{\xi^{0}}$.

Proof. We can assume without loss of generality that $K$ is small so that

$$
\begin{aligned}
& \left\{\lambda_{\xi^{0}, j}^{+}\left(\eta^{\prime}\right) ; 1 \leq j \leq l^{\prime} \text { and } \eta^{\prime} \in K\right\} \cap\left\{\lambda_{\xi_{0}^{0}, j}\left(\eta^{\prime}\right) ; 1 \leq j \leq r_{m_{\xi^{0}}}-l^{\prime}\right. \\
& \text { and } \left.\eta^{\prime} \in K\right\}=\varnothing \quad \text { if } \xi_{n}^{0} \in \mathbb{R} \text { and } r_{m \xi^{0}} \neq 0
\end{aligned}
$$

(see Lemma 2.5). Let $\xi_{n}^{0} \in \boldsymbol{R}$ and $\mathscr{C}_{\xi^{0}, j}^{+1}\left(1 \leq j<t\left(\xi^{0 \prime}\right), t^{\prime}\left(\xi^{0}\right) \leq j \leq s\left(\xi^{0}\right)\right)$ be simple closed curves enclosing only the roots with positive imaginary part of $P_{j, \xi_{0}}\left(\eta^{\prime} ; \lambda\right)=0$ for $\eta^{\prime} \in K$ (see Lemma 2.4). Let $\mathscr{C}_{\xi^{0}, 0}^{+}$be a simple closed curve enclosing only the roots $\lambda_{\xi^{0}, j}^{+}\left(\eta^{\prime}\right), 1 \leq j \leq l^{\prime}$, of $P_{\xi^{0}}\left(\eta^{\prime}, \lambda\right)=0$ for $\eta^{\prime} \in K$ if $r_{m_{\xi_{0}}} \neq 0$ and $\mathscr{C}_{\xi^{\prime}}^{+}$a simple closed curve enclosing only the roots with positive imaginary part of $P_{\xi^{0}, q}(\eta ; \lambda)=0$ for $\eta^{\prime} \in K$ (see Lemma 2.7). From the relations between the roots of $P\left(\nu^{-1} \xi^{\prime \prime}+\eta^{\prime}, \lambda\right)=0$ and

+ $\partial M$ denotes the boundary of $M$.

it The $\lambda_{j}^{+}\left(\xi^{\prime}\right)$ are continuous and $\operatorname{Im} \lambda_{j}^{+}\left(\xi^{\prime}-i \gamma \vartheta^{\prime}\right)>0$ for $\xi^{\prime} \in \mathbb{R}^{n-1}$ and $r_{\mathbb{R}^{n}}>_{r_{0}}$. 
the roots of $P_{j, \xi^{0}}\left(\eta^{\prime} ; \lambda\right)=0, P_{\xi^{0}}\left(\eta^{\prime}, \lambda\right)=0$ and $P_{\xi^{0}, q}(\eta ; \lambda)=0$ there exists $\nu_{K}^{\prime}(>0)$ such that $\left\{\lambda_{j}^{+}\left(\nu^{-1} \xi^{0 \prime}+\eta^{\prime}\right)\right\}_{1 \leq j \leq l} \cap\left\{\lambda_{j}^{-}\left(\nu^{-1} \xi^{0 \prime}+\eta^{\prime}\right)\right\}_{1 \leq j \leq m^{\prime}-l}=\varnothing$ for $\eta^{\prime} \in K, 0<\nu \leq \nu_{K}^{\prime}$. So we can take $\mathscr{C}_{\nu}^{+}$to be a simple closed curve enclosing only the roots $\lambda_{j}^{+}\left(\nu^{-1} \xi^{0 \prime}+\eta^{\prime}\right), 1 \leq j \leq l$, of $P\left(\nu^{-1} \xi^{0 \prime}+\eta^{\prime}, \lambda\right)=0$ for $\eta^{\prime}$ $\in K, 0<\nu \leq \nu_{K}^{\prime}$. For $\eta^{\prime} \in K$ and $0<\nu \leq \nu_{K}^{\prime}$ we have

$$
\begin{aligned}
& \sigma^{k}\left(\nu^{-1} \xi^{0 \prime}+\eta^{\prime}\right)=(2 \pi i)^{-1} \int_{\mathscr{E}_{\nu}^{+}} \lambda^{k} \partial / \partial \xi_{n} P\left(\nu^{-1} \xi^{0 \prime}+\eta^{\prime}, \lambda\right) \\
& \times P\left(\nu^{-1} \xi^{0 \prime}+\eta^{\prime}, \lambda\right)^{-1} d \lambda \\
& =\sum_{j=1}^{t-1}(2 \pi i)^{-1} \int_{\mathscr{F}_{\left(\xi 0^{\prime \prime}, 0\right), j}^{+}} \lambda^{k} \partial / \partial \xi_{n} P\left(\nu^{-1} \xi^{0 \prime}+\eta^{\prime}, \nu^{-1 / l_{j}} \lambda\right) \\
& \times\left(P_{l_{j},\left(\xi 0^{\prime}, 0\right)}\left(\eta^{\prime}, 0 ; \lambda\right)+0(1)\right)^{-1} \nu^{m-(k+1) / l_{j}-m\left(\xi \xi^{\prime}, 0\right)\left(l_{j}\right)} d \lambda \\
& +(2 \pi i)^{-1} \int_{\mathscr{\mho}_{\xi o}^{+}} \lambda^{k} \partial / \partial \xi_{n} P\left(\nu^{-1} \xi^{0 \prime}+\eta^{\prime}, \nu^{-1} \lambda\right) \\
& \times\left(P_{\xi^{0}, q}\left(\eta^{\prime}, 0 ; \lambda\right)+o(1)\right)^{-1} \nu^{m-q-k-1} d \lambda \\
& +\sum_{\xi_{n}^{0} \in \boldsymbol{R}, \partial^{q} / \partial \xi_{1}^{q} P^{0}\left(\xi^{0}, \xi_{n}^{0}\right)=0}\left[\sum_{j=t^{\prime}\left(\xi^{0}\right)}^{s\left(\xi^{0}\right)}(2 \pi i)^{-1}\right. \\
& \times \int_{\mathscr{E}_{\xi 0, j}^{+}}\left(\nu^{-1} \xi_{n}^{0}+\nu^{-1 / l_{j}} \lambda\right)^{k} \partial / \partial \xi_{n} P\left(\nu^{-1} \xi^{0 \prime}+\eta^{\prime}, \nu^{-1} \xi_{n}^{0}+\nu^{-1 / l_{j}} \lambda\right) \\
& \times\left(P_{l_{j, \xi^{0}}}\left(\eta^{\prime}, 0 ; \lambda\right)+o(1)\right)^{-1} \nu^{m-1 / l_{j}-m_{\xi 0}\left(l_{j}\right)} d \lambda \\
& +\left(1-\delta_{0 r_{m_{\xi} 0}(\xi 0)}\right) \times(2 \pi i)^{-1} \int_{\mathcal{E}_{\xi^{0}}^{+}, 0}\left(\nu^{-1} \xi_{n}^{0}+\lambda\right)^{k} \\
& \times \partial / \partial \xi_{n} P\left(\nu^{-1} \xi^{0 \prime}+\eta^{\prime}, \nu^{-1} \xi_{n}^{0}+\lambda\right) \\
& \left.\times\left(P_{\xi^{0}}\left(\eta^{\prime}, \lambda\right)+o(1)\right)^{-1} \nu^{m-m_{\xi^{0}}} d \lambda\right],
\end{aligned}
$$

where each $O(1)$ is a polynomial of $\eta^{\prime}, \lambda$ and $\nu^{1 / L}$ and vanishes for $\nu=0$ and $L$ is a positive integer. So there exists $\nu_{K}(>0)$ such that each integrand in (2.20) can be expanded in a power series of $\nu^{1 / L}$, which converges uniformly in $\eta^{\prime} \in K$ and $0 \leq \nu \leq \nu_{K}$. From this the lemma easily follows.

Q.E.D.

Lemma 2.9. Let $1 \leq k \leq l$. For any compact set $K$ in $\mathbb{R}^{n-1}-i \dot{\Gamma}_{\xi^{0}}$, there exist $\nu_{K}$ and $r_{K}(>0)$ such that $\sigma^{k}\left(\nu^{-1} r \xi^{\prime \prime}+r \eta^{\prime}\right)$ is well-defined 
when $r_{K} \eta^{\prime} \in \mathbb{R}^{n-1}-i \gamma_{0} \vartheta^{\prime}-i \dot{\Gamma}_{\xi^{\prime}}, \alpha \eta^{\prime} \in K$ for some $\alpha \in \mathbb{C} \quad(|\alpha|=1), 0<\nu$ $\leq \nu_{K}$ and $r \geq r_{K}$. We have

$$
\begin{aligned}
& \left(\nu r^{-1}\right)^{s_{k}} \sigma^{k}\left(\nu^{-1} r \xi^{0 \prime}+r \eta^{\prime}\right)=\sum_{j=0}^{\infty} \sum_{i=0}^{\infty} r^{q_{k j}} \sigma_{\xi^{\prime}, j}^{k i}\left(\eta^{\prime}\right) \nu^{j / L} r^{-i}, \\
& \sigma_{\xi^{\prime}, j}^{k 0}\left(\eta^{\prime}\right) \not \equiv 0 \text { if } \sigma_{\xi^{\prime}, j}^{k}\left(\eta^{\prime}\right) \not \equiv 0,
\end{aligned}
$$

whose convergence is uniform in $\left\{\left(\eta^{\prime}, \nu, r\right) ; r_{K} \eta^{\prime} \in \mathbb{R}^{n-1}-i \gamma_{0} \vartheta^{\prime}-i \dot{\Gamma}_{\xi 0 \%}\right.$ $\alpha \eta^{\prime} \in K$ for some $\alpha \in C(|\alpha|=1), 0 \leq \nu \leq \nu_{K}$ and $\left.r \geq r_{K}\right\}$, where the $q_{k j}$ are rational numbers. Moreover the $\sigma_{\xi^{\prime}, j}^{k i}\left(\eta^{\prime}\right)$ are holomorphic in $\mathbb{R}^{n-1}-i \dot{\Gamma}_{\xi \circ}$ and homogeneous and

$$
\sigma_{\xi 0^{\prime}, j}^{k}\left(r \eta^{\prime}\right)=r^{q_{k j}+j / L} \sum_{i=0}^{\infty} \sigma_{\xi 0^{\prime}, j}^{k i}\left(\eta^{\prime}\right) r^{-i},
$$

whose convergence is uniform in $\left\{\left(\eta^{\prime}, r\right) ; r_{K} \eta^{\prime} \in \mathbb{R}^{n-1}-i \gamma_{0} \vartheta^{\prime}-i \dot{\Gamma}_{\xi 0}, \alpha \eta^{\prime}\right.$ $\in K$ for some $\alpha \in \mathbb{C}(|\alpha|=1)$ and $\left.r \geq r_{K}\right\}$.

Proof. Modifying the curves $\mathscr{C}_{\nu}^{+}, \mathscr{C}_{\left.\xi^{0}, 0\right), j}^{+}, \mathscr{C}_{\xi^{0}}^{+}$, and $\mathscr{C}_{\xi^{0}, j}^{+}$in the proof of Lemma 2.8, we have

$$
\begin{aligned}
& \sigma^{k}\left(\nu^{-1} r \xi^{0 \prime}+r \eta^{\prime}\right)=\left(2 \pi_{i}\right)^{-1} \int_{\mathscr{C}_{\nu}^{+}} \lambda^{k} \partial / \partial \xi_{n} P\left(\nu^{-1} r \xi^{0 \prime}+r \eta^{\prime}, \lambda\right) \\
& \times P\left(\nu^{-1} r \xi^{\prime \prime}+r \eta^{\prime}, \lambda\right)^{-1} d \lambda \\
& =\left[\sum_{j=1}^{t-1}(2 \pi i)^{-1} \int_{\mathscr{E}_{\left(\xi^{\prime}, 0\right), j}^{+}} \lambda^{k} \partial / \partial \xi_{n} P\left(\nu^{-1} r \xi^{0 \prime}+r \eta^{\prime}, \nu^{-1 / l} j r \lambda\right)\right. \\
& \times\left(P_{\left.l_{j,\left(5^{\prime}\right.}, 0\right)}^{0}\left(\eta^{\prime}, 0 ; \lambda\right)+0(1)\right)^{-1} \nu^{m-(k+1) / l_{j}-m\left(\xi^{\prime}, 0\right)\left(l_{j}\right)} d \lambda \\
& +(2 \pi i)^{-1} \int_{\mathscr{E}_{\xi^{0}}^{+},} \lambda^{k} \partial / \partial \xi_{n} P\left(\nu^{-1} r \xi^{0 \prime}+r \eta^{\prime}, \nu^{-1} r \lambda\right) \\
& \times\left(P_{\xi 0^{\prime}, q}^{0}\left(\eta^{\prime}, 0 ; \lambda\right)+o(1)\right)^{-1} \nu^{m-k-1-q} d \lambda \\
& +\sum_{\xi_{n}^{0} \in \mathbb{R}, \partial^{q} / \partial \hat{\xi}_{1}^{q} P^{0}\left(\xi^{0}, \xi_{n}^{0}\right)=0}\left\{\sum_{j=t^{\prime}\left(\xi_{5}^{0}\right)}^{s\left(\xi_{0}^{0}\right)}(2 \pi i)^{-1}\right. \\
& \times \int_{\mathscr{\xi}_{\xi^{0}, j}^{+}}\left(\nu^{-1} \xi_{n}^{0}+\nu^{-1 / l_{j}} \lambda\right)^{k} \\
& \times \partial / \partial \xi_{n} P\left(\nu^{-1} r \xi^{0 \prime}+r \eta^{\prime}, \nu^{-1} r \xi_{n}^{0}+\nu^{-1 / l_{j}} r \lambda\right) \\
& \times\left(P_{l_{j}, \xi^{0}}^{0}\left(\eta^{\prime}, 0 ; \lambda\right)+0(1)\right)^{-1} \nu^{m-1 / l_{j}-1 n_{\xi^{0}}\left(l_{j}\right)} d \lambda
\end{aligned}
$$




$$
\begin{aligned}
& +\left(1-\delta_{0 r_{\xi_{\xi}}(\xi 0)}\right) \times(2 \pi i)^{-1} \int_{\mathscr{\varepsilon}_{\xi^{0}, 0}^{+}}\left(\nu^{-1} \xi_{n}^{0}+\lambda\right)^{k} \\
& \times \partial / \partial \xi_{n} P\left(\nu^{-1} r \xi^{0 \prime}, \nu^{-1} r \xi_{n}^{0}+r \lambda\right)\left(P_{\xi^{0}}^{0}\left(\eta^{\prime}, \lambda\right)+o(1)\right)^{-1} \\
& \left.\left.\times \nu^{m-m_{\xi^{0}}} d \lambda\right\}\right] \times r^{-m+k+1},
\end{aligned}
$$

where each $O(1)$ is a polynomial of $\eta^{\prime}, \lambda, \nu^{1 / L}$ and $r^{-1}$ and vanishes for $\nu=0, r^{-1}=0$. In fact, for example, we have

$$
\begin{aligned}
&\left(\nu r^{-1}\right) m\left(\nu^{-1} r \xi^{0 \prime}+r \eta^{\prime}, \nu^{-1} r \xi_{n}^{0}+\nu^{-1 / l_{j}} r \lambda\right) \\
&=\left(\nu r^{-1}\right)^{m_{\xi^{0}\left(l_{j}\right)}} P_{l_{j}, \xi^{0}}\left(r \eta^{\prime}, 0 ; r^{\left(l_{j}-1\right) / l_{j}} \lambda\right) \\
& \quad \quad \sum_{n_{h}>\xi_{\xi^{0}\left(l_{j}\right)}}\left(\nu r^{-1}\right)^{n_{h}} P_{l_{j}, \xi^{0}, h}\left(r \eta^{\prime}, 0 ; r^{\left(l_{j}-1\right) / l_{j}} \lambda\right) \\
& \operatorname{deg}_{l_{j}} P_{l_{j}, \xi^{0}, h}\left(\eta^{\prime}, 0 ; \lambda\right) \leq n_{h} .
\end{aligned}
$$

Therefore we have

$$
\begin{aligned}
& \left(\nu r^{-1}\right)^{m} P\left(\nu^{-1} r \xi^{0 \prime}+r \eta^{\prime}, \nu^{-1} r \xi_{n}^{0}+\nu^{-1 / l_{j}} r \lambda\right) \\
& \quad=\nu^{m_{\xi^{0}\left(l_{j}\right)}}\left(P_{l_{j}, \xi^{0}}^{0}\left(\eta^{\prime}, 0 ; \lambda\right)+0(1)\right) \quad \text { as } \nu, r^{-1} \rightarrow 0 .
\end{aligned}
$$

So there exist $\nu_{K}$ and $r_{K}(>0)$ such that each integrand in (2.21) can be expanded in a power series of $\nu^{1 / L}$ and $r^{-1}$, which converges uniformly in $\left\{\left(\eta^{\prime}, \nu, r\right) ; \eta^{\prime} \in K, r \in \mathbb{C}, \nu \in C,|r| \geq r_{K}\right.$ and $\left.0 \leq|\nu| \leq \nu_{K}\right\}$. We note that

$$
\sigma_{\xi 0, j}^{k i}\left(\alpha \eta^{\prime}\right)=\alpha^{q_{k j}+j / L-i} \sigma_{\xi 0}^{k i}, j\left(\eta^{\prime}\right)
$$

when $\alpha \eta^{\prime}, \eta^{\prime} \in \boldsymbol{R}^{n-1}-i \dot{\Gamma}_{\xi^{0}}$, where $1^{q_{k j}+j / L-i}=1$. This completes the proof.

Q.E.D.

Let us consider $\dot{\Gamma}_{\xi^{0} \%}$ Although $\Gamma\left(P_{(0,1)}\right)=\Gamma\left(\left(P_{(0,1)}\right)_{\left(\xi^{0} \%, 0\right)}\right)$ does not always hold, we can prove the inner semi-continuity of $\dot{\Gamma}_{\xi^{\prime}}$.

Lemma 2. 10. Let $\xi^{0^{\prime}} \in \boldsymbol{R}^{n-1}$ and assume that $0<\rho<l_{1}\left(\xi^{\prime \prime}, 0\right)$. Then for any compact set $\widetilde{M}$ in $\Gamma\left(\left(P_{(0,1)}\right)_{(\xi 0,0)}\right)$ there exist a neighborhood $U$ of $\xi^{\prime \prime}$ and positive numbers $r_{0}$, $t_{0}$ such that

$$
P\left(r \xi^{\prime}-i r t \eta^{\prime}-i \gamma_{0} \vartheta^{\prime}, r^{1 / \rho} \lambda-i r t \eta_{n}\right) \neq 0
$$

when $\eta \in \widetilde{M}, \xi^{\prime} \in U, \lambda \in \boldsymbol{R},|\lambda| \geq 1, r \geq r_{0}$ and $0<t \leq t_{0}$.

Proof. Put 


$$
\begin{array}{r}
f\left(\nu, t, \zeta^{\prime}, \lambda, s, t, \eta\right)=P\left(\nu^{-1} r \xi^{0 \prime}+r \zeta^{\prime}-i r t \eta^{\prime}-i\left(s+\gamma_{0}\right) \vartheta^{\prime},\right. \\
\left.\nu^{-1 / \rho} r^{1 / \rho} \lambda-i r t \eta_{n}\right),
\end{array}
$$

where $0<\nu \leq \nu_{0}, r \geq r_{0}, \quad \zeta^{\prime} \in \mathbb{R}^{n-1}, \quad\left|\zeta^{\prime}\right| \leq \varepsilon, \quad$ Re $s \geq 0$, Re $t \geq 0,|s| \leq s_{0},|t|$ $\leq t_{0}, \lambda \in \mathbb{R},|\lambda| \geq 1$ and $\eta \in \widetilde{M}$. Then we have

$$
\begin{aligned}
& \left(\nu r^{-1}\right)^{m} f\left(\nu, r, \zeta^{\prime}, \lambda, s, t, \eta\right) \\
& =\left(\nu r^{-1}\right)^{m_{\rho}\left(\xi^{\prime}, 0\right)} P_{\rho,\left(\xi^{\circ}, 0\right)}\left(r \zeta^{\prime}-i r t \eta^{\prime}-i\left(s+\gamma_{0}\right) \vartheta^{\prime},-i r t \eta_{n} ; \lambda\right) \\
& \quad+\sum_{n_{h}>m_{\rho}\left(\xi^{\prime}, 0\right)}\left(\nu r^{-1}\right)^{n_{h}} P_{\rho,\left(\xi^{\prime}, 0\right), h}\left(r \zeta^{\prime}-i r t \eta^{\prime}-i\left(s+\gamma_{0}\right) \vartheta^{\prime},-i r t \eta_{n} ; \lambda\right), \\
& \operatorname{deg}_{\rho} P_{\rho,\left(\xi^{\circ}, 0\right), h}(\eta ; \lambda) \leq n_{h} .
\end{aligned}
$$

Since $0<\rho<l_{1}$,

$$
P_{\rho,\left(\xi^{\circ}, 0\right)}(\eta ; \lambda)=\left(P_{m}\right)_{\xi_{0},}\left(\eta^{\prime}\right) \lambda^{m^{\prime}} .
$$

Since the degree of $P_{\rho,\left(\hat{\xi}^{0}, 0\right), h}(\eta ; \lambda)$ with respect to $\lambda$ is not greater than $m$, it follows that

$$
\begin{aligned}
& \nu^{m-m_{\rho}\left(\xi^{\prime}, 0\right)} r^{-m+m^{\prime}(\rho-1) / \rho} \lambda^{-m^{\prime}} f\left(\nu, r, \zeta^{\prime}, \lambda, s, t, \eta\right) \\
& \quad=\left(P_{m^{\prime}}^{0}\right)_{\xi^{0}}\left(\zeta^{\prime}-i t \eta^{\prime}-i r^{-1}\left(s+\gamma_{0}\right) \vartheta^{\prime}\right)+0(1) \text { as } \nu, r^{-1} \rightarrow 0,
\end{aligned}
$$

i.e. for any positive number $\delta$ there exist $r_{0}, \nu_{0}(>0)$ such that

$$
\begin{aligned}
& \mid \nu^{m-m_{\rho}\left(\xi^{0}, 0\right)} r^{-m+m^{\prime}(\rho-1) / \rho} \lambda^{-m^{\prime}} f\left(\nu, r, \zeta^{\prime}, \lambda, s, t, \eta\right) \\
& \quad-\left(P_{m^{\prime}}^{0}\right)_{\xi^{\prime}}\left(\zeta^{\prime}-i t \eta^{\prime}-i r^{-1}\left(s+\gamma_{0}\right) \vartheta^{\prime}\right) \mid<\delta
\end{aligned}
$$

when $0<\nu \leq \nu_{0}, r \geq r_{0},\left|\zeta^{\prime}\right| \leq \varepsilon,|s| \leq s_{0},|\lambda| \geq 1,|t| \leq t_{0}$ and $\eta \in \widetilde{M}$. So we can apply the same argument as in Lemma 3.7 in [7] to $f\left(\nu, r, \zeta^{\prime}, \lambda, s, t, \eta\right)$ and we obtain the lemma.

Q.E.D.

Lemma 2. 11. Let $\xi^{0 \prime} \in \mathbb{R}^{n-1}$ and $M$ be a compact set in $\dot{\Gamma}_{\xi \circ}$. There exists a neighborhood $U$ of $\xi^{\prime \prime}$ such that

$$
M \subset \dot{\Gamma}_{\xi^{\prime}} \quad \text { for } \xi^{\prime} \in U
$$

Remark. From the proof of Lemma 2.11 it follows that

$$
\bigcup_{\xi_{n} \in \mathbb{R}} \Gamma\left(P_{\left(\xi^{0}, \xi_{n}\right)}\right) \supset \Gamma\left(\left(P_{(0,1)}\right)_{\left(\hat{\xi}^{0}, 0\right)}\right) \text {. }
$$


Proof. Assume that there exists a sequence $\left\{\xi^{j}, \eta^{j \prime}\right\}_{j=1,2, \ldots}$ such that $\left|\xi^{j \prime}-\xi^{0 \prime}\right|<1 / j, \xi_{n}^{j} \in \mathbb{R}, \eta^{j \prime} \in M$ and $P_{\xi^{j}}^{0}\left(-i \eta^{j \prime}, 0\right)=0$. Then from the inner semi-continuity of $\dot{\Gamma}_{\xi}$ (or $\Gamma_{\xi}$ ) it follows that $\left|\xi_{n}^{j}\right| \rightarrow \infty$ as $j \rightarrow \infty$. Let $\widetilde{M}$ be a compact set in $\Gamma\left(\left(P_{(0,1)}\right)_{\left(\xi^{0}, 0\right)}\right)$ such that the interior of $\widetilde{M}$ includes $M \times\{0\}$. Lemma 2.10 implies that there exist a neighborhood $U$ of $\xi^{0 \prime}$ and $\lambda_{0}, t_{0}(>0)$ such that

$$
P^{0}\left(\xi^{\prime}-i t \eta^{\prime}, \lambda-i t \eta_{n}\right) \neq 0
$$

when $\eta \in \widetilde{M}, \xi^{\prime} \in U, \lambda \in \mathbb{R},|\lambda| \geq \lambda_{0}$ and $0<t \leq t_{0}$, which leads us to a contradiction, using Rouché's theorem.

Q.E.D.

\section{$\S$ 3. Proof of Theorem 1 . 1}

Let $P(\xi)$ be written in the form

$$
P(\xi)=\prod_{j=1}^{q} p_{j}(\xi)^{\nu_{j}}
$$

where the $p_{j}(\xi)$ are irreducible polynomials. We assume that $\prod_{j=1}^{q^{\prime}} p_{j}\left(\xi^{\prime}\right.$, $\lambda)^{\nu_{j}}=0$ has roots $\lambda_{1}^{+}\left(\xi^{\prime}\right), \cdots, \lambda_{l}^{+}\left(\xi^{\prime}\right)$ when $\xi^{\prime} \in \mathbb{R}^{n-1}-i \gamma_{0} \vartheta^{\prime}-i \Gamma_{0}$, i.e. $\prod_{j=q^{\prime}+1}^{q} p_{j}\left(\xi^{\prime}, \lambda\right)^{\nu_{j}}=0$ does not have roots with positive imaginary part when $\xi^{\prime} \in \mathbb{R}^{n-1}-i \gamma_{0} \vartheta^{\prime}-i \Gamma_{0}$. Then put

$$
\dot{\Gamma}_{\xi^{\prime}}=\bigcap_{j=1}^{q^{\prime}}\left\{\underset{\xi_{n}^{0} \in \mathbb{R}}{\Gamma} \dot{\Gamma}\left(\left(p_{j}\right)_{\xi^{0}}\right) \cap \dot{\Gamma}\left(\left(\left(p_{j}\right)_{(0,1)}\right)_{\left(\xi^{\prime} 0^{\prime}, 0\right)}\right)\right\} .
$$

We note that

$$
\left(\left(p_{j}\right)_{(0,1)}\right)_{\left(\xi^{0} \%, 0\right)}(\eta)=\left(\left(p_{j}\right)_{(0,1)}\right)_{\left(\xi^{0}, \xi_{n}\right)}(\eta) \quad \text { for all } \xi_{n} \in \mathbb{R} .
$$

The following lemma is obvious.

Lemma 3. 1. $\oint B_{j}\left(\xi^{\prime}, \lambda\right) \lambda^{k-1} P_{+}\left(\xi^{\prime}, \lambda\right)^{-1} d \lambda$ is a polynomial of $\xi^{\prime}$ and $\sigma^{k}\left(\xi^{\prime}\right), 1 \leq k \leq l$, when $P_{+}\left(\xi^{\prime}, \lambda\right)$ is rell-defined.

From Lemma 2. 8 we have the following

Lemma 3. 2. Let $\xi^{0 \prime} \in \mathbb{R}^{n-1}$. For any compact set $K$ in $\mathbb{R}^{n-1}$ -ir $9^{\prime}-i \dot{\Gamma}_{\xi^{0}}$, there exists $\nu_{K}(>0)$ such that $R\left(\nu^{-1} \xi^{0 \prime}+\eta^{\prime}\right)$ is welldefined for $\eta^{\prime} \in K$ and $0<\nu \leq \nu_{K}$ and 


$$
\begin{aligned}
& \nu^{h_{\xi^{0}}} R\left(\nu^{-1} \xi^{0 \prime}+\eta^{\prime}\right)=\sum_{j=0}^{\infty} \nu^{j / L} R_{\xi^{0}, j}\left(\eta^{\prime}\right), \\
& R_{\xi^{0}, 0}\left(\eta^{\prime}\right) \equiv R_{\xi^{0} \circ}\left(\eta^{\prime}\right) \not \equiv,
\end{aligned}
$$

whose convergence is uniform in $\left(\eta^{\prime}, \nu\right) \in K \times\left\{0 \leq \nu \leq \nu_{K}\right\}$, where $h_{\xi_{0} 0}$, is a rational number and $L$ is a positive integer. Moreover the $R_{\xi^{0}, j}\left(\eta^{\prime}\right)$ are holomorphic in $\mathbb{R}^{n-1}-i \gamma_{0} \vartheta^{\prime}-i \dot{\Gamma}_{\hat{s}^{0}}$.

Remark. $\quad R_{\xi^{0}, 0}\left(\eta^{\prime}\right) \equiv R_{\xi^{0}}\left(\eta^{\prime}\right)$ is the localization of $R\left(\xi^{\prime}\right)$ at $\xi^{0 \prime}$. Moreover this lemma for $\xi^{\prime \prime}=0$ implies that $R\left(\xi^{\prime}\right)$ is holomorphic in $\boldsymbol{R}^{n-1}-i \gamma_{0} \vartheta^{\prime}-i \dot{\Gamma} \quad$ (see [3]).

The following lemma is also obtained by Lemma 2.9.

Lemma 3. 3. Let $\xi^{0 \prime} \in \mathbb{R}^{n-1}$. For any compact set $K$ in $\mathbb{R}^{n-1}$ $-i \dot{\Gamma}_{\xi 0 \%}$, there exist $\nu_{K}$ and $r_{K}(>0)$ such that $R\left(\nu^{-1} r \xi^{0 \prime}+r \eta^{\prime}\right)$ is welldefined rohen $r_{K} \eta^{\prime} \in \mathbb{R}^{n-1}-i \gamma_{0} \vartheta^{\prime}-i \dot{\Gamma}_{5^{\prime}}, \alpha \eta^{\prime} \in K$ for some $\alpha \in \mathbb{C}(|\alpha|=1)$, $0<\nu \leq \nu_{K}$ and $r \geq r_{K}$. We have

$$
\begin{aligned}
& \left(\nu r^{-1}\right)^{h_{\xi^{\prime}}} R\left(\nu^{-1} r \xi^{0 \prime}+r \eta^{\prime}\right)=\sum_{j=0}^{\infty} \sum_{i=0}^{\infty} r^{h_{j}^{\left(\xi^{0} 0^{\prime}\right)-i} \nu^{j / L}} R_{\xi^{\prime} 0^{\prime}, j}^{i}\left(\eta^{\prime}\right), \\
& R_{\xi^{0}, j}^{0}\left(\eta^{\prime}\right) \not \equiv 0 \text { if } R_{\hat{\xi}^{\prime}, j}\left(\eta^{\prime}\right) \neq 0,
\end{aligned}
$$

rohose convergence is uniform in $\left\{\left(\eta^{\prime}, \nu, r\right) ; r_{K} \eta^{\prime} \in \mathbb{R}^{n-1}-i \gamma_{0}{ }^{\prime}-i \dot{\Gamma}_{\xi 0 \%}\right.$, $\alpha \eta^{\prime} \in K$ for some $\alpha \in \mathbb{C}(|\alpha|=1), 0 \leq \nu \leq \nu_{K}$ and $\left.r \geq r_{K}\right\}$, where the $h_{j}\left(\xi^{0 \prime}\right)$ are rational numbers. Moreover the $R_{5^{\prime \prime}, j}^{i}\left(\eta^{\prime}\right)$ are holomorphic in $\mathbb{R}^{n-1}-i \dot{\Gamma}_{\text {so }^{0}}$ and homogeneous and

$$
R_{\xi^{\prime}, j}\left(r \eta^{\prime}\right)=r^{h_{j}\left(5^{\prime} 0^{\prime}\right)+j / L} \sum_{i=0}^{c_{0}} r^{-i} R_{\xi^{0}, j}^{i}\left(\eta^{\prime}\right),
$$

whose convergence is uniform in $\left\{\left(\eta^{\prime}, r\right) ; r_{K} \eta^{\prime} \in \mathbb{R}^{n-1}-i \gamma_{0} \theta^{\prime}-i \dot{\Gamma}_{\xi^{\circ}}, a \cdot \eta^{\prime}\right.$ $\in K$ for some $\alpha \in C(|\alpha|=1)$ and $\left.r \geq r_{K}\right\}$.

Remark. The principal part $\left(R_{\xi_{0} 0}\right)^{0}\left(\eta^{\prime}\right)$ of $R_{\hat{\varepsilon}^{0}}\left(\eta^{\prime}\right)$ is equal to $R_{\xi^{\prime \prime}, 0}^{0}\left(\eta^{\prime}\right)$. Moreover this lemma for $\xi^{0 \prime}=0$ implies Lemma 3.2 in [3].

In the above two lemmas we can replace $R\left(\xi^{\prime}\right)$ by $R_{j k}\left(\xi^{\prime}\right)$ or $P_{ \pm}\left(\xi^{\prime}, \lambda\right)$ with obvious modifications. 
Lemma 3. 4. Let $\xi^{0} \in \mathbb{R}^{n}$. There exist the localizations $P_{ \pm \xi^{0}}(\xi)$ and $\left(P_{ \pm}^{0}\right)_{\xi^{0}}(\xi)$ of $P_{ \pm}(\xi)$ and $P_{ \pm}^{0}(\xi)$ at $\xi^{0}$, respectively, and

$$
P_{ \pm \xi^{0}}^{0}(\xi) \equiv\left(P_{ \pm}^{0}\right)_{\xi^{0}}(\xi)=\left(P_{ \pm \xi^{0}}\right)^{0}(\xi) .
$$

Proof. The existence of $P_{ \pm \xi^{0}}(\xi),\left(P_{ \pm}^{0}\right)_{\xi^{0}}(\xi)$ and $\left(P_{ \pm \xi^{0}}\right)^{0}(\xi)$ follows from Lemmas 3.2 and 3.3 and the above remark. It easily follows that $P_{\xi^{0}}(\xi)=P_{+\xi^{0}}(\xi) P_{-\xi^{0}}(\xi), \quad\left(P_{\xi^{0}}\right)^{0}(\xi)=\left(P^{0}\right)_{\xi^{0}}(\xi)=\left(P_{+}^{0}\right)_{\xi^{0}}(\xi)\left(P_{-}^{0}\right)_{\xi^{0}}(\xi)$ and $\operatorname{deg}^{\dagger}\left(P_{ \pm \xi^{0}}\right)^{0}(\xi) \leq \operatorname{deg}\left(P_{ \pm}^{0}\right)_{\xi^{0}}(\xi)$. This implies (3.1). Q.E.D.

Let us denote by $\Gamma\left(R_{\xi_{0}}\right)$ the component of the set $\left\{\eta^{\prime} \in \dot{\Gamma}_{\xi^{0}} ;\left(R_{\xi 0},\right)^{0}\right.$ $\left.\left(-i \eta^{\prime}\right) \neq 0\right\}$ which contains $\vartheta^{\prime \dagger \dagger}$. Then we have the following

Lemma 3.5 ([8]). Let $\xi^{0 \prime} \in \mathbb{R}^{n-1} . \quad \Gamma\left(R_{\xi^{\prime}}\right)$ is an open convex cone and

$$
\begin{array}{ll}
R_{\xi^{\circ}}\left(\xi^{\prime}\right) \neq 0 & \text { for } \xi^{\prime} \in \mathbb{R}^{n-1}-i \gamma_{1} \vartheta^{\prime}-i \Gamma\left(R_{\xi^{\prime}}\right), \\
\left(R_{\xi^{\prime}}\right)^{0}\left(\xi^{\prime}\right) \neq 0 & \text { for } \xi^{\prime} \in \mathbb{R}^{n-1}-i \Gamma\left(R_{\xi^{\prime}}\right) .
\end{array}
$$

Let us denote by $\Gamma\left(P_{+\xi^{0}}\right)$ the component of the set $\left\{\eta \in \dot{\Gamma}_{\xi^{0}} \times \mathbb{R}\right.$; $\left.P_{+\xi_{0}^{0}}^{0}(-i \eta) \neq 0\right\}$ which contains $\theta$. Then we have also the following

Lemma 3. 6. Let $\xi^{0} \in \boldsymbol{R}^{n} . \Gamma\left(P_{+\xi_{0}}\right)$ is an open convex cone and

$$
\begin{aligned}
& P_{+\xi^{0}}(\xi) \neq 0 \quad \text { for } \xi \in \mathbb{R}^{n}-i \gamma_{0} \theta-i \Gamma\left(P_{+\xi^{0}}\right), \\
& P_{+\xi^{0}}^{0}(\xi) \neq 0 \quad \text { for } \xi \in \mathbb{R}^{n}-i \Gamma\left(P_{+\xi^{0}}\right), \\
& \Gamma\left(P_{+\xi^{0}}\right) \supset \Gamma_{\xi^{0}} .
\end{aligned}
$$

In our case we can prove Lemma 3.2 in [8].

Lemma 3. 7. Let $\xi^{0 \prime} \in \mathbb{R}^{n-1}$ and let $M$ be a compact set in $\dot{\Gamma}_{\xi^{0}}$. Then there exist a conic neighborhood $\Delta_{1}\left(\subset \mathbb{R}^{n-1}\right)$ of $\xi^{0 \prime}$ and positive numbers $C, t_{0}$ such that $P_{+}\left(\zeta^{\prime}, \lambda\right)$ is holomorphic in $\left(\zeta^{\prime}, \lambda\right) \in A \times \mathbb{C}$, where

$$
\begin{array}{r}
\Lambda=\left\{\zeta^{\prime}=\xi^{\prime}-i t\left|\xi^{\prime}\right| \eta^{\prime}-i \gamma_{0} \vartheta^{\prime} ; \xi^{\prime} \in \Delta_{1},\left|\xi^{\prime}\right| \geq C, \eta^{\prime} \in M I\right. \\
\\
\text { and } \left.0<t \leq t_{0}\right\} .
\end{array}
$$

\footnotetext{
$+\operatorname{deg} p^{0}(\xi)$ denotes the degree of homogeneity of $p^{0}$.

it $\left(R_{\xi 0},\right)^{0}\left(-i \vartheta^{\prime}\right) \neq 0$ was shown in [7].
} 
Therefore $R\left(\zeta^{\prime}\right)$ and $R_{j k}\left(\zeta^{\prime}\right)$ are also holomorphic in $\Lambda$.

Proof. The lemma is trivial for $\xi^{\prime \prime}=0$. So we assume that $\xi^{\prime \prime}$ $\in \mathbb{R}^{n-1} \backslash\{0\}$. Let $\lambda\left(\zeta^{\prime}\right)$ be a root of $p_{j}\left(\zeta^{\prime}, \lambda\right)=0$. We can assume that $\lambda\left(\zeta^{\prime}\right)$ is continuous in $\Lambda$ when $C$ and $t_{0}$ are suitably chosen. In fact, there exist a conic neighborhood $\Delta_{1}\left(\subset \mathbb{R}^{n-1}\right)$ of $\xi^{\prime \prime}$ and $C, t_{0}(>0)$ such that

$$
\begin{array}{r}
p_{j(0,1)}\left(\xi-i t|\xi| \eta-i \gamma_{0} \vartheta\right) \neq 0 \text { if } \xi^{\prime} \in \Delta_{1},\left|\xi^{\prime}\right| \geq C, \eta^{\prime} \in M \\
\text { and } 0<t \leq t_{0} .
\end{array}
$$

For $p_{j(0,1)}(\xi)$ is independent of $\xi_{n}$ and $M \subset \dot{\Gamma}_{\xi^{0}} \subset \dot{\Gamma}\left(\left(p_{j(0,1)}\right)_{\left(\xi \xi^{0}, 0\right)}\right)$. The argument in Section 2 shows that $\lim _{\nu \rightarrow 0} \nu \lambda\left(\nu^{-1} \xi^{0 \prime}-i \eta^{\prime}-i \gamma_{0} \vartheta^{\prime}\right)=\mu_{0}$ exists if $\lim _{\nu \rightarrow 0}\left|\operatorname{Im} \nu \lambda\left(\nu^{-1} \xi^{0 \prime}-i \eta^{\prime}-i \gamma_{0} \vartheta^{\prime}\right)\right|=0$, where $\eta^{\prime} \in M$. Moreover from Lemmas 2.4 and 2.7 it follows that $\mu_{0}$ is a real root of $\partial^{q} / \partial \xi_{1}^{a} p_{j}^{0}\left(\xi^{0 \prime}, \lambda\right)=0$. Now let us assume that $\mu_{0}$ is a real multiple root of $\partial^{q} / \partial \xi_{1}^{q} p_{j}^{0}\left(\xi^{0 \prime}, \lambda\right)=0$. We can assume without loss of generality that $M$ is small so that $\left\{\left(\eta^{\prime}, \eta_{n}\right)\right.$; $\eta^{\prime} \in M$ and $\left.\eta_{n}^{1} \leq \eta_{n} \leq \eta_{n}^{2}\right\} \subset \Gamma\left(p_{j\left(\xi^{0}, \mu_{0}\right)}\right)$ for some $\eta_{n}^{1}, \eta_{n}^{2} \in \mathbb{R}$. Then it follows that there exist a conic neighborhood $\tilde{\Delta}$ of $\left(\xi^{0 \prime}, \mu_{0}\right)$ and $C, t_{0}(>0)$ such that

$$
\begin{aligned}
& p_{j}\left(\xi^{\prime}-i t\left|\xi^{\prime}\right| \eta^{\prime}-i \gamma_{0} \vartheta^{\prime}, \lambda-i t\left|\xi^{\prime}\right| \eta_{n}\right) \neq 0 \\
& \quad \text { if }\left(\xi^{\prime}, \lambda\right) \in \tilde{\Delta},\left|\xi^{\prime}\right| \geq C, \eta^{\prime} \in M, \eta_{n}^{1} \leq \eta_{n} \leq \eta_{n}^{2} \text { and } 0<t \leq t_{0} .
\end{aligned}
$$

This implies that

$$
\operatorname{Im} \lambda\left(\xi^{\prime}-i t\left|\xi^{\prime}\right| \eta^{\prime}-i \gamma_{0} \vartheta^{\prime}\right) \notin\left[-i t\left|\xi^{\prime}\right| \eta_{n}^{2},-t\left|\xi^{\prime}\right| \eta_{n}^{1}\right]
$$

for $\xi^{\prime} \in \Delta_{1},\left|\xi^{\prime}\right| \geq C, \eta^{\prime} \in M$ and $0<t \leq t_{0}$, modifying $\Delta_{1}, C$ and $t_{0}$, if necessary (see Lemma 3.2 in [8]). If $\theta^{\prime} \notin M$, we choose a continuous curve $\eta^{\prime}(\theta)$ in $\dot{\Gamma}_{\xi^{0}}$, such that $\eta^{\prime}(0)=\theta^{\prime}$ and $\eta^{\prime}(1) \in M$ and we repeat the above argument for each small neighborhood of $\eta^{\prime}(\theta), 0 \leq \theta \leq 1$. This proves the lemma (see Lemma 3.2 in [8]).

Q.E.D.

Put

$$
t_{j} \equiv t_{j}\left(\xi^{0 \prime}\right)=h_{\xi^{0}}+h_{j}\left(\xi^{0 \prime}\right),
$$

where $h_{\xi^{\circ}}$ and $h_{j}\left(\xi^{0 \prime}\right)$ are defined in Lemma 3. 3. Then it is easy to see 
that $t_{j}\left(\xi^{0 \prime}\right)$ is an integer and that $t_{j}\left(\xi^{0 \prime}\right) \leq t_{0}(0)^{\dagger}$. Put

$$
t \equiv \ell\left(\xi^{0 \prime}\right)=\max t_{j}\left(\xi^{0 \prime}\right), \omega \equiv \omega\left(\xi^{0 \prime}\right)=\min \left\{j ; t\left(\xi^{0 \prime}\right)=t_{j}\left(\xi^{0 \prime}\right)\right\} .
$$

It easily follows that $t\left(\xi^{0 \prime}\right)=t_{0}(0)$. From Lemma 3.3 we have the following

Lemma 3. 8. Let $\xi^{0 \prime} \in \mathbb{R}^{n-1}$. The principal part $R^{0}\left(\xi^{\prime}\right)$ of $R\left(\xi^{\prime}\right)$ is rell-defined and there exists the localization $\left(R^{0}\right)_{\xi^{\circ}}\left(\eta^{\prime}\right)$ of $R^{0}\left(\xi^{\prime}\right)$ at $\xi^{0 \prime}$. Moreover for any compact set $K$ in $\mathbb{R}^{n-1}-i \dot{\Gamma}_{\xi^{0}}$ there exists $\nu_{K}(>0)$ such that

$$
\begin{aligned}
& \nu^{h_{\xi^{0}}-t_{0}(0)-\omega\left(\xi^{\prime}\right) / L} R^{0}\left(\xi^{0 \prime}+\nu \eta^{\prime}\right) \\
& \quad=\sum_{t_{j}\left(\xi_{0} 0^{\prime}\right)=t_{0}(0)} \nu^{\left(j-\omega\left(\xi^{0}\right)\right) / L} R_{\xi^{0}, j}^{0}\left(\eta^{\prime}\right),
\end{aligned}
$$

whose convergence is uniform in $\left\{\left(\eta^{\prime}, \nu\right) ; \eta^{\prime} \in \mathbb{R}^{n-1}-i \dot{\Gamma}_{\xi^{\circ}}, \alpha \eta^{\prime} \in K\right.$ for some $\alpha \in \mathbb{C}(|\alpha|=1)$ and $\left.0 \leq \nu \leq \nu_{K}\right\}$, and

$$
\left(R^{0}\right)_{\xi_{0} 0}\left(\eta^{\prime}\right)=R_{\xi_{0 \prime}^{\prime}, \omega\left(\xi^{\prime}\right)}^{0}\left(\eta^{\prime}\right) \text {. }
$$

Let $\Gamma\left(\left(R^{0}\right)_{\xi^{0}}\right)$ be the component of the set $\left\{\eta^{\prime} \in \dot{\Gamma}_{\xi^{0}} ;\left(R^{0}\right)_{\xi^{0}}\left(-i \eta^{\prime}\right)\right.$ $\neq 0\}$ which contains $\vartheta^{\prime \prime \dagger}$.

Lemma 3.9 ([8]). Let $\xi^{\prime \prime} \in \mathbb{R}^{n-1} . \quad \Gamma\left(\left(R^{0}\right)_{\xi^{\prime}}\right)$ is an open convex cone and

$$
\left(R^{0}\right)_{\xi^{0}}\left(\xi^{\prime}\right) \neq 0 \text { for } \xi^{\prime} \in \boldsymbol{R}^{n-1}-i \Gamma\left(\left(R^{0}\right)_{\xi^{0}}\right) .
$$

Lemma 3. 10 ([8]). Let $\xi^{0 \prime} \in \mathbb{R}^{n-1}$. For any compact set $M$ in $\Gamma\left(\left(R^{0}\right)_{\xi^{0}}\right)$, there exist a conic neighborhood $\Delta_{1}\left(\subset \mathbb{R}^{n-1}\right)$ of $\xi^{0 \prime}$ and positive numbers $C, t_{0}$ such that

$$
\begin{array}{r}
R\left(\xi^{\prime}-i t\left|\xi^{\prime}\right| \eta^{\prime}-i \gamma_{1} \theta^{\prime}\right) \neq 0 \text { if } \eta^{\prime} \in M, \xi^{\prime} \in \Delta_{1},\left|\xi^{\prime}\right| \geq C \\
\text { and } 0<t \leq t_{0} .
\end{array}
$$

Let $\tilde{\xi}^{0} \in \mathbb{R}^{n+1}$ and put

${ }^{\dagger} R\left(r \eta^{\prime}\right)=r^{t_{0}(0)} \sum_{i=0}^{\infty} r^{-i} R_{\hat{\xi}(0,0}^{i}\left(\eta^{\prime}\right)$.

tt $\left(R^{0}\right)_{\xi^{0}},\left(-i \vartheta^{\prime}\right) \neq 0$ was shown in [8]. 


$$
\begin{gathered}
\Gamma_{\tilde{\xi}^{0}}=\left\{\tilde{\eta} \in \mathbb{R}^{n+1} ;\left(\eta^{\prime}, \eta_{n+1}\right) \in \Gamma\left(P_{\left(\xi^{0}, \xi_{n+1}^{0}\right)}\right)\right\} \\
\cap\left(\Gamma\left(P_{+\xi^{0}}\right) \times \mathbb{R}^{R}\right) \cap\left(\Gamma\left(R_{\xi^{0}}\right) \times \mathbb{R}^{2}\right), \\
\Gamma_{\tilde{\xi} 0}^{0}=\left\{\tilde{\eta} \in \mathbb{R}^{n+1} ;\left(\eta^{\prime}, \eta_{n+1}\right) \in \Gamma\left(P_{\left(\xi^{0}, \hat{\xi}_{n+1}^{0}\right)}\right)\right\} \\
\cap\left(\Gamma\left(P_{+\xi^{0}}\right) \times \mathbb{R}\right) \cap\left(\Gamma\left(\left(R^{0}\right)_{\xi^{0}}\right) \times \mathbb{R}^{2}\right) .
\end{gathered}
$$

Then Theorem 1.1 can be proved by the same arguments as in [7], [8]. (1. 2) follows from Lemma 4.1.

\section{$\S$ H. Some Remarks and Examples}

Lemma 4. $\mathbb{1}([8])$. Let $\xi^{0 \prime} \in \mathbb{R}^{n-1} . \quad \Gamma\left(\left(R^{0}\right)_{\hat{\xi}^{0}}\right) \subset \Gamma\left(R_{\xi^{0}}\right)$.

Let us prove the inner semi-continuity of $\Gamma\left(\left(R^{0}\right)_{\xi^{\prime}}\right)$ and, therefore, $\Gamma_{\tilde{\xi}}^{0}$.

Lemma 4. 2. Let $\xi^{0 \prime} \in \mathbb{R}^{n-1}$ and let $M$ be a compact set in $\Gamma\left(\left(R^{0}\right)_{\hat{\xi}^{\circ}}\right)$. Then there exist a neighborhood $U$ of $\xi^{\prime \prime}$ and positive number $t_{0}$ such that $R^{0}\left(\xi^{\prime}\right)$ is holomorphic in $U-i D$ and $R^{0}\left(\xi^{\prime}\right) \neq 0$ for $\xi^{\prime} \in U-i D$, where $D=\left\{t \eta^{\prime} ; \eta^{\prime} \in M^{\circ} \text { and } 0<t \leq t_{0}\right\}^{\dagger}$.

Proof. We can assume without loss of generality that $P(\xi)$ is irreducible. Since $M \subset \dot{\Gamma}\left(\left(P_{(0,1)}\right)_{\left(\xi^{0}, 0\right)}\right)$, it follows that there exist a neighborhood $U$ of $\xi^{\prime \prime}$ and $t_{0}, \nu_{0}(>0)$ such that

$$
P_{(0,1)}\left(\nu^{-1} \xi\right)=P_{m^{\prime}}\left(\nu^{-1} \xi^{\prime}\right) \neq 0
$$

if $\xi^{\prime} \in U-i D-i \nu_{1} \gamma_{0} \vartheta^{\prime}, 0<\nu \leq \nu_{1} \leq \nu_{0}$. Let $K$ be a compact set in $U-i D$. Then there exists $\nu_{K}(>0)$ such that $\nu_{K} \leq \nu_{0}$ and $K \subset U-i D-i \nu_{K} \gamma_{0} \theta^{\prime}$. Let $\lambda_{j}^{ \pm}\left(\xi^{\prime} ; \nu\right)$ be a root of $P\left(\nu^{-1} \xi^{\prime}, \nu^{-1} \lambda\right)=0$ such that $\lambda_{j}^{ \pm}\left(\xi^{\prime} ; \nu\right)=\nu \lambda_{j}^{ \pm}\left(\nu^{-1} \xi^{\prime}\right)$ when $\xi^{\prime} \in K$ and $0<|\nu| \leq \nu_{K}$. In fact, since $P_{m^{\prime}}\left(\nu^{-1} \xi^{\prime}\right) \neq 0$ for $\xi^{\prime} \in K$ and $0<|\nu| \leq \nu_{K}$, modifying $\nu_{K}$ if necessary, the above statement is meaningful. Moreover we can assume that $\lambda_{j}^{ \pm}\left(\xi^{\prime} ; \nu\right)$ is continuous when $\xi^{\prime} \in K$ and $0 \leq|\nu| \leq \nu_{K}$. Since $\lambda_{j}^{ \pm}\left(\xi^{\prime} ; 0\right)$ is a root of $P^{0}\left(\xi^{\prime}, \lambda\right)=0$, the same argument as in Lemma 3.7 gives

\footnotetext{
† $\stackrel{\circ}{M}$ denotes the interior of $M$.
} 


$$
\left\{\lambda_{j}^{+}\left(\xi^{\prime} ; 0\right)\right\} \cap\left\{\lambda_{j}^{-}\left(\xi^{\prime} ; 0\right)\right\}=\varnothing \quad \text { for } \xi^{\prime} \in K,
$$

modifying $U$ and $t_{0}$ if necessary. Therefore it follows from cntinuity of $\lambda_{j}^{ \pm}\left(\xi^{\prime} ; \nu\right)$ that

$$
\left\{\lambda_{j}^{+}\left(\xi^{\prime} ; \nu\right)\right\} \cap\left\{\lambda_{j}^{-}\left(\xi^{\prime} ; \nu\right)\right\}=\varnothing \quad \text { for } \xi^{\prime} \in K \text { and }|\nu| \leq \nu_{K},
$$

modifying $\nu_{K}$ if necessary. Put

$$
\begin{aligned}
& P_{+}\left(\xi^{\prime}, \lambda ; \nu\right)=\prod_{j=1}^{l}\left(\lambda-\lambda_{j}^{+}\left(\xi^{\prime} ; \nu\right)\right)=\lambda^{l}+b_{1}^{+}\left(\xi^{\prime} ; \nu\right) \lambda^{l-1}+\cdots+b_{l}^{+}\left(\xi^{\prime} ; \nu\right), \\
& P_{+}\left(\xi^{\prime}, \lambda\right)=\prod_{j=1}^{l}\left(\lambda-\lambda_{j}^{+}\left(\xi^{\prime}\right)\right)=\lambda^{l}+a_{1}^{+}\left(\xi^{\prime}\right) \lambda^{l-1}+\cdots+a_{l}^{+}\left(\xi^{\prime}\right) .
\end{aligned}
$$

(4. 1) implies that the $b_{j}^{+}\left(\xi^{\prime} ; \nu\right)$ are holomorphic in $\left\{\left(\xi^{\prime}, \nu\right) ; \xi^{\prime} \in K\right.$ and $\left.|\nu| \leq \nu_{K}\right\}$. Moreover we have $a_{j}^{+}\left(\nu^{-1} \xi^{\prime}\right)=\nu^{-j} b_{j}^{+}\left(\xi^{\prime} ; \nu\right)$. Therefore we have

$$
b_{j}^{+}\left(\xi^{\prime} ; \nu\right)=a_{j 0}^{+}\left(\xi^{\prime}\right)+\nu a_{j 1}^{+}\left(\xi^{\prime}\right)+\nu^{2} a_{j 2}^{+}\left(\xi^{\prime}\right)+\cdots,
$$

whose convergence is uniform in $\left\{\left(\xi^{\prime}, \mu\right) ; \xi^{\prime} \in K\right.$ and $\left.|\nu| \leq \nu_{K}\right\} . \quad a_{j k}^{+}\left(\xi^{\prime}\right)$ is holomorphic in $U-i D$ and homogeneous of degree $j-k$. So $R^{0}\left(\xi^{\prime}\right)$ is well-defined and holomorphic in $U-i D$. (3.2) and the above result yields us

$$
R^{0}\left(\xi^{\prime}\right) \neq 0 \quad \text { for } \xi^{\prime} \in U-i D,
$$

using the same argument as in the proof of Lemma 3.7 in [8].

Q.E.D.

Theorem 4. 3. Let $\xi^{0 \prime} \in \boldsymbol{R}^{n-1}$ and let $M$ be a compact set in $\Gamma\left(\left(R^{0}\right)_{\xi_{0}}\right)$. There exists a neighborhood $U$ of $\xi^{0 \prime}$ such that $M \subset \Gamma\left(\left(R^{0}\right)_{\xi^{\prime}}\right) \quad$ for $\xi^{\prime} \in U$.

Proof. It is obvious that $M \subset \dot{\Gamma}_{\xi^{\prime}}$ for $\xi^{\prime} \in U$, shrinking $U$. Now assume that there exist $\xi^{1 \prime} \in U$ and $\eta^{0 \prime} \in M$ such that $\left(R^{0}\right)_{\xi^{1}}\left(-i \eta^{\prime \prime}\right)=0$, where $U$ is sufficiently small. Since $\left(R^{0}\right)_{\xi^{1}}\left(-i \eta^{\prime}\right) \not \equiv 0$, there exists $\zeta^{0 \prime}$ $\in \mathbb{C}^{n-1}$ such that $\xi^{1 \prime}-i\left(\eta^{0 \prime}+\mu \zeta^{0 \prime}\right) \in U-i M$ for $|\mu| \leq 1$ and $\left(R^{0}\right)_{\xi^{1}}\left(-i\left(\eta^{0 \prime}\right.\right.$ $\left.\left.+\zeta^{\prime \prime}\right)\right) \neq 0$. Therefore it follows that there exist $\varepsilon, \delta(>0)$ such that

$$
\left|\left(R^{0}\right)_{\xi^{1}}\left(-i\left(\eta^{0 \prime}+\mu \zeta^{0 \prime}\right)\right)\right| \geq 2 \varepsilon \quad \text { for }|\mu|=\delta .
$$


On the other hand from (3.2) we have

$$
\begin{aligned}
& \mid t^{h_{\xi_{1}^{\prime}-t_{0}(0)-\omega\left(\xi^{\prime \prime}\right) / L}} R^{0}\left(\xi^{1 \prime}-i t\left(\eta^{0 \prime}+\mu \zeta^{0 \prime}\right)\right) \\
& \quad-\left(R^{0}\right)_{\xi^{\prime}}\left(-i\left(\eta^{0 \prime}+\mu \zeta^{0 \prime}\right)\right) \mid<\varepsilon \text { for }|\mu|=\delta \text { and } 0<t \leq t_{1} \quad\left(\leq t_{0}\right),
\end{aligned}
$$

where $t_{0}$ and $t_{1}$ are suitably chosen. Rouché's theorem implies that $R^{0}\left(\xi^{1 \prime}-i t\left(\eta^{0 \prime}+\mu \zeta^{0 \prime}\right)\right)$ has zeros within $|\mu|<\delta$ for $0<t \leq t_{1}$, which is a contradiction to Lemma 4. 2 .

Q.E.D.

Theorem 4.3 yields us the following

Theorem A. A. $\bigcup_{\tilde{\xi} \in \mathbb{R}^{n+1} \backslash\{0\}} K_{\tilde{\xi}}^{0} \times\{\tilde{\xi}\}$ is closed in $T^{*} X \backslash 0$.

In Section 2 the developments of $\sigma^{k}\left(\nu^{-1} \xi^{0 \prime}+\eta^{\prime}\right)$ and $\sigma^{k}\left(\nu^{-1} r \xi^{0 \prime}+r \eta^{\prime}\right)$ was given. However we can similarly obtain the developments $f\left(\nu^{-1} \xi^{\prime \prime}+\eta^{\prime}\right)$ and $f\left(\nu^{-1} r \xi^{0 \prime}+r \eta^{\prime}\right)$, where

$$
f\left(\xi^{\prime}\right)=(2 \pi i)^{-1} \int_{\mathcal{q}^{+}} g\left(\xi^{\prime}, \lambda\right) P\left(\xi^{\prime}, \lambda\right)^{-1} d \lambda
$$

and $g\left(\xi^{\prime}, \lambda\right)$ is a polynomial of $\left(\xi^{\prime}, \lambda\right)$ and $\mathscr{C}^{+}$encloses only the roots $\lambda_{1}^{+}\left(\xi^{\prime}\right), \cdots, \lambda_{l}^{+}\left(\xi^{\prime}\right)$ of $P\left(\xi^{\prime}, \lambda\right)=0$. This will be useful for hyperbolic systems.

Next let us consider some examples.

Example 4. 5. Put $n=4$ and

$$
\begin{aligned}
& P(\xi)=\left(\xi_{1}^{2}-\xi_{2}^{2}-\xi_{3}^{2}-\xi_{4}^{2}+a \xi_{3}\right)\left(\xi_{1}^{2}-\xi_{4}^{2}\right), \quad a>0, \\
& B_{1}(\xi)=1, B_{2}(\xi)=\left(-\xi_{1}-i \xi_{3}\right) \xi_{4}-\xi_{4}^{2} .
\end{aligned}
$$

Then we have $R\left(\xi^{\prime}\right)=i \xi_{3}+\sqrt[t]{\xi_{1}^{2}-\xi_{2}^{2}-\xi_{3}^{2}+a \xi_{3}}$. It is obvious that $\left\{P, B_{1}\right.$, $\left.B_{2}\right\}$ satisfies the condition (A). We can show that $\underset{\tilde{\xi} \in \mathbb{R}^{5}\{\{0\}}{\bigcup} K_{\tilde{\xi}} \times\{\tilde{\xi}\}$ is not closed in $T^{*} X \backslash 0$ and that

$$
\begin{aligned}
& \bigcup_{\tilde{\xi} \in \mathbb{R}^{5} \backslash\{0\}} \bigcup_{j=0}^{\infty} \operatorname{supp} \widetilde{F}_{\tilde{\xi}, j} \times\{\tilde{\xi}\}=\bigcup_{\tilde{\xi} \in \mathbb{R}^{5} \backslash\{0\}} K_{\tilde{\xi}} \times\{\tilde{\xi}\} \\
& \subsetneq W F(\widetilde{F}) \subset W F_{A}(\widetilde{F}) \subset \bigcup_{\tilde{\xi} \in \mathbb{R}^{5} \backslash\{0\}} K_{\tilde{\xi}}^{0} \times\{\tilde{\xi}\}
\end{aligned}
$$

(see [9]). Moreover we have

$$
\overline{\operatorname{ch}}\left[\left.W F(\widetilde{F})\right|_{\tilde{\xi}}\right]=\overline{\operatorname{ch}}\left[\left.W F_{A}(\widetilde{F})\right|_{\tilde{\xi}^{0}}\right]=K_{\tilde{\xi} 0}^{0} \quad \text { for } \quad \widetilde{\xi}^{0} \neq 0 \text {. }
$$


Example 4. 6. Put $n=3$ and

$$
\begin{aligned}
& P(\xi)=\left(\left(\xi_{1}-\xi_{2}\right)^{2}-\xi_{3}^{2}+a\right)\left(\left(2 \xi_{1}-\xi_{2}\right)^{2}-\xi_{3}^{2}\right), \\
& B_{1}(\xi)=1, \quad B_{2}(\xi)=\xi_{3} .
\end{aligned}
$$

Then $R\left(\xi^{\prime}\right)=-1$ and $\left\{P, B_{1}, B_{2}\right\}$ satisfies the condition (A). We note that $\left(\xi_{1}-\xi_{2}\right)^{2}-\xi_{3}^{2}+a$ is irreducible when $a \neq 0$. It is easy to see that

$$
\begin{array}{r}
\left.W F(\widetilde{F})\right|_{(1,1,-1,1)}=\{\widetilde{z} \in X ; \widetilde{z}=\alpha(2,-1,0,-1)+\beta(2,-1,1,0) \\
+\gamma(1,-1,0,0), \alpha, \beta>0 \text { and } \gamma \geq 0\} \text { when } a \neq 0, \\
\left.W F(\widetilde{F})\right|_{(1,1,-1,1)}=\{\widetilde{z} \in X ; \widetilde{z}=\alpha(2,-1,0,-1)+\beta(2,-1,1,0) \\
\text { and } \alpha, \beta>0\} \text { when } a=0 .
\end{array}
$$

This shows that so called lateral wave appears when $a \neq 0$.

In conclusion, the author wishes to thank Professor M. Matsumura for his valuable advices and helpful discussions.

\section{References}

[1] Atiyah M. F., Bott, R. and Gårding. L., Lacunas for hyperbolic differential operators with constant coefficients. I, Acta Math., 124 (1970), 109-189.

[2] Garnir, H. G., Solution élémentaire des problèms aux limites hyperboliques, to appear.

[3] Sakamoto, R., $\mathcal{E}$-well posedness for hyperbolic mixed problems with constant coefficients, J. Math. Kyoto Univ., 14 (1974), 93-118.

[4] Shibata, Y., A characterization of the hyperbolic mixed problems in a qurter space for differential oprators with costant coefficients, Publ. RIMS, Kyoto Unjv., 15 (1979), 357-399.

[5] Svensson, S. L., Necessary and sufficient conditions for the hyperbolicity of polinomials with hyperbolic principal part Ark. Mat., 8 (1970), 145-162.

[6] Tsuji, M., Fundamental solutions of hyperbolic mixed problems with constant coefficients, Proc. Japan Acad., 51 (1975), 369-373.

[7] Wakabayashi, S., Singularities of the Riemann functions of hyperbolic mixed problems in a quarter-space, Publ. RIMS. Kyoto Univ., 11 (1976), 417-440.

[8] - Analytic wave front sets of the Riemann functions of hyperbolic mixed problems in a quarter-space, Publ. RIMS, Kyoto Univ., 11 (1976), 785-807.

[9] - Propagation of singularities for hyperbolic mixed problems, Proc. NATO Advanced Study Institute, Reidel, 1976. 